\title{
Therapy-induced lipid uptake and remodeling underpin ferroptosis hypersensitivity in prostate cancer
}

Kaylyn D. Tousignant ${ }^{1 \dagger}$, Anja Rockstroh ${ }^{1}$, Berwyck L. J. Poad ${ }^{3}$, Ali Talebi', Reuben S. E. Young ${ }^{3}$, Atefeh Taherian Fard ${ }^{1}$, Rajesh Gupta ${ }^{3}$, Tuo Zang ${ }^{1}$, Chenwei Wang ${ }^{1}$, Melanie L. Lehman ${ }^{1,5}$, Johan V. Swinnen ${ }^{4}$, Stephen J. Blanksby ${ }^{3}$, Colleen C. Nelson ${ }^{1}$ and Martin C. Sadowski ${ }^{1,2^{*}}$ (i)

\begin{abstract}
Background: Metabolic reprograming, non-mutational epigenetic changes, increased cell plasticity, and multidrug tolerance are early hallmarks of therapy resistance in cancer. In this temporary, therapy-tolerant state, cancer cells are highly sensitive to ferroptosis, a form of regulated cell death that is caused by oxidative stress through excess levels of iron-dependent peroxidation of polyunsaturated fatty acids (PUFA). However, mechanisms underpinning therapy-induced ferroptosis hypersensitivity remain to be elucidated.

Methods: We used quantitative single-cell imaging of fluorescent metabolic probes, transcriptomics, proteomics, and lipidomics to perform a longitudinal analysis of the adaptive response to androgen receptor-targeted therapies (androgen deprivation and enzalutamide) in prostate cancer (PCa).

Results: We discovered that cessation of cell proliferation and a robust reduction in bioenergetic processes were associated with multidrug tolerance and a strong accumulation of lipids. The gain in lipid biomass was fueled by enhanced lipid uptake through cargo non-selective (macropinocytosis, tunneling nanotubes) and cargo-selective mechanisms (lipid transporters), whereas de novo lipid synthesis was strongly reduced. Enzalutamide induced extensive lipid remodeling of all major phospholipid classes at the expense of storage lipids, leading to increased desaturation and acyl chain length of membrane lipids. The rise in membrane PUFA levels enhanced membrane fluidity and lipid peroxidation, causing hypersensitivity to glutathione peroxidase (GPX4) inhibition and ferroptosis. Combination treatments against AR and fatty acid desaturation, lipase activities, or growth medium supplementation with antioxidants or PUFAs altered GPX4 dependence.

(Continued on next page)
\end{abstract}

\footnotetext{
* Correspondence: martin.sadowski@qut.edu.au

${ }^{\dagger}$ Kaylyn D. Tousignant and Martin C. Sadowski contributed equally to this work.

${ }^{1}$ Australian Prostate Cancer Research Centre - Queensland, Institute of Health and Biomedical Innovation, School of Biomedical Sciences, Faculty of Health, Queensland University of Technology (QUT), Princess Alexandra Hospital, Translational Research Institute, Brisbane, Australia

${ }^{2}$ Cancer \& Ageing Research Program, Institute of Health and Biomedical Innovation, School of Biomedical Sciences, Faculty of Health, Queensland University of Technology (QUT), Translational Research Institute, Brisbane, Australia

Full list of author information is available at the end of the article
}

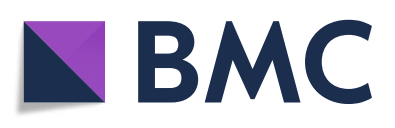

(c) The Author(s). 2020 Open Access This article is licensed under a Creative Commons Attribution 4.0 International License, which permits use, sharing, adaptation, distribution and reproduction in any medium or format, as long as you give appropriate credit to the original author(s) and the source, provide a link to the Creative Commons licence, and indicate if changes were made. The images or other third party material in this article are included in the article's Creative Commons licence, unless indicated otherwise in a credit line to the material. If material is not included in the article's Creative Commons licence and your intended use is not permitted by statutory regulation or exceeds the permitted use, you will need to obtain permission directly from the copyright holder. To view a copy of this licence, visit http://creativecommons.org/licenses/by/4.0/ The Creative Commons Public Domain Dedication waiver (http://creativecommons.org/publicdomain/zero/1.0/) applies to the data made available in this article, unless otherwise stated in a credit line to the data. 
(Continued from previous page)

Conclusions: Our work provides mechanistic insight into processes of lipid metabolism that underpin the acquisition of therapy-induced GPX4 dependence and ferroptosis hypersensitivity to standard of care therapies in PCa. It demonstrates novel strategies to suppress the therapy-tolerant state that may have potential to delay and combat resistance to androgen receptor-targeted therapies, a currently unmet clinical challenge of advanced PCa. Since enhanced GPX4 dependence is an adaptive phenotype shared by several types of cancer in response to different therapies, our work might have universal implications for our understanding of metabolic events that underpin resistance to cancer therapies.

Keywords: Therapy resistance, Multidrug tolerance, Metabolic reprograming, Lipid remodeling, Lipid uptake, Ferroptosis, GPX4, Prostate cancer

\section{Background}

Despite significant advancements in detection and treatment over the past decades, prostate cancer $(\mathrm{PCa})$ remains the second most commonly diagnosed cancer among men and the third leading cause of cancer mortality in men worldwide [1]. A major challenge in PCa management stems not from the lack of initial treatment options, but in therapy adaptation leading to resistance. Given the dependence of $\mathrm{PCa}$ cells on androgens for growth, proliferation, survival, and differentiation, the primary treatment for men with advanced $\mathrm{PCa}$ is androgen deprivation therapy (ADT), which is being increasingly supplemented with a new generation of potent androgen synthesis inhibitors (abiraterone) or androgen receptor (AR) antagonists bicalutamide, enzalutamide, and apalutamide [2,3], hereafter cumulatively termed androgen-targeted therapies (ATTs). Despite initial treatment response, unfortunately ATTs will ultimately fail in most $\mathrm{PCa}$ patients, and disease progresses to castrate-resistant PCa (CRPC) [3, 4] in which PCa cells have often adopted a number of complex resistance mechanisms, including AR amplification, mutation, or splice variants to maintain AR function in the low androgen environment $[3,5]$. Other resistance mechanisms bypass AR by exploiting alternative signaling and metabolic pathways. Understanding the early underlying molecular mechanisms that ultimately result in resistance to ATTs is critical for the discovery and development of co-treatment strategies and the definition of therapeutic windows to extend the efficacy of ATTs and combat therapy resistance.

While previous studies in $\mathrm{PCa}$ provided an extensive characterization of PCa models with established ATT resistance [6-8], therapy-induced early metabolic reprogramming that underpins therapy survival and development of drug resistance has remained largely unexplored. Recent work in breast, melanoma, lung, and ovarian cancer showed that different targeted anti-cancer therapies induced a transient quiescent state of negligible growth termed drug-induced persister cells [9]. Persister cells are characterized by increased expression of markers of dedifferentiation, enhanced lipid peroxidation, an acquired dependency on lipid hydroperoxidase GPX4, and sensitivity to ferroptosis that offer novel therapeutic strategies to combat resistance [9]. Whether ATTs cause PCa cells to acquire the persister cell phenotype remains to be shown.

Ferroptosis is a form of regulated cell death characterized by iron-dependent lipid peroxidation of polyunsaturated fatty acids (PUFAs) that was recently identified as a metabolic vulnerability in several types of cancer, including lymphoma, renal cell carcinoma, and breast cancer $[10,11]$. In particular, reactive oxygen speciesmediated peroxidation of PUFAs arachidonic acid and adrenic acid within the phospholipid class of phosphatidylethanolamines are potent activators of ferroptosis [12]. Glutathione peroxidase 4 (GPX4), a selenocysteine enzyme that utilizes glutathione to reduce toxic lipid peroxides into non-toxic lipid alcohols, is critical for the protection against lipid peroxide-mediated damage and ferroptosis induction [11]. Recently, a second, glutathioneindependent protective pathway containing ferroptosissuppressor-protein- 1 (FSP1/AIFM2) was found to act in parallel to GPX4 [13, 14]. FSP1 functions as an oxidoreductase with coenzyme Q10 at the plasma membrane to generate lipophilic radical-trapping antioxidants that halt lipid peroxidation [13]. While ferroptosis is an attractive and highly promising concept to combat resistance to multiple therapies in various types of cancer, the metabolic changes that lead to enhanced ferroptosis sensitivity in the context of therapy resistance remain uncharacterized [9].

Dysregulation of lipid homeostasis is a metabolic hallmark associated with $\mathrm{PCa}$ tumorigenesis and disease progression to CRPC in response to ATTs. Yet, therapyinduced metabolic reprograming and lipid supply mechanisms other than de novo lipid synthesis have not been studied in this context. Previous data from our longitudinal xenograft model of progression to CRPC demonstrated alterations in lipid metabolism during the development to CRPC [15], including increased intratumoral levels of essential PUFAs derived from dietary sources implicating lipid uptake. Emerging evidence 
reveals lipid scavenging routes utilized by cancer cells during stress in cell culture and pre-clinical models, including lipid transporters [16], the formation of tunneling nanotubes [17], serum albumin-lipid conjugate uptake, and macropinocytosis of necrotic cell debris from the microenvironment [18]. Many of these lipid supply pathways converge in lysosomes, where degradation of endocytic vesicles releases lipids and other nutrients intracellularly [19], highlighting the importance of lysosomal activity for lipid supply.

In the present study, we delineated the longitudinal metabolic changes in PCa cells in response to ATTs that lead to therapy-induced persister cells characterized by increased lipid content and uptake and substantial lipid remodeling, multidrug tolerance and ferroptosis hypersensitivity. We show that acquisition of this therapyinduced phenotype can be efficiently suppressed by several combination treatments directed against lipid processing enzymes. In addition, this work identified enhanced dependence of persister cell on lysosomal processing of exogenous cholesterol. Together, our work gained novel mechanistic insight into therapy-induced ferroptosis hypersensitivity and provided multiple strategies that have the potential to extend the efficacy of ATTs and delay resistance development and disease progression to lethal CRPC.

\section{Methods}

\section{Cell culture}

LNCaP (ATCC, CVCL_0395) and C4-2B (ATCC, CVCL_4784) cells were cultured in RPMI medium (Thermo Fisher) supplemented with 5\% FBS. Medium was changed every 3 days and cells were incubated at $37{ }^{\circ} \mathrm{C}$ in $5 \% \mathrm{CO}_{2}$. For treatments representing androgendeprivation therapy, cells were cultured in RPMI medium supplemented with $5 \%$ charcoal -stripped serum (CSS). For AR-targeted therapies, cells were cultured in $5 \%$ or $10 \%$ FBS with AR antagonist enzalutamide (Enz, $10 \mu \mathrm{M})$. Cells were passaged at approximately $80 \%$ confluency by trypsinization. Cell lines were genotyped in March 2018 by Genomics Research Centre (Brisbane) and routinely tested for mycoplasma infection.

\section{RNA extraction and quantitative real-time polymerase chain reaction ( $q R T-P C R$ )}

Forty-eight hours after seeding, cells were treated with the indicated inhibitors. After an additional $48 \mathrm{~h}$, RNA was harvested using the RNEasy mini kit (Qiagen) following the manufacturer's instructions. Before extraction, RNA was treated with DNase (Qiagen) to improve RNA purity. Concentration of RNA was measured using a NanoDrop ND-1000 Spectrophotometer (ThermoScientific), and cDNA was prepared from $2 \mu \mathrm{g}$ total RNA with Superscript III (Invitrogen). qRT-PCR was performed with SYBR Green PCR Master Mix (Invitrogen) on a ViiA-7 Real-Time PCR system (Applied Biosystems). Determination of relative mRNA levels was calculated using the comparative $\Delta \Delta \mathrm{CT}$ method [20] compared to the expression of housekeeping gene receptor like protein 32 (RPL32) in each treatment and calculated as fold change relative to vehicle control (DMSO). All experiments were performed in triplicate, and analysis and statistics were performed with GraphPad Prism software. Primer sequences can be found in supplementary information (Table S5).

\section{Microarray gene expression profiling on the $180 \mathrm{k}$ VPC custom arrays}

For gene expression profiling, triplicates of each sample were analyzed on a custom 180k Agilent oligo microarray (VPCv3, ID032034, GEO: GPL16604). This array contains probes mapping to human protein-coding as well as non-coding loci, with probes targeting exons, 3' UTRs, 5'UTRs, intronic, and intergenic regions. RNA was isolated using the RNeasy Mini Kit (Qiagen, Hilden, Germany) according to the manufacturer's protocol including an on-column DNAse treatment step. RNA purity and quality were evaluated on a NanoDrop1000 (Thermo Fisher Scientific Inc., Waltham, USA) and Agilent 2100 Bioanalyzer (Agilent Technologies, Santa Clara, USA). One hundred fifty nanograms of RNA of each sample was amplified and labeled using the Agilent "Low Input Quick Amp Labeling Kit" for One-Color Microarray-Based Gene Expression Analysis. In brief, the input RNA was reverse transcribed into cDNA, using an oligo-dT/T7-promoter hybrid primer which introduces a T7 promoter region into the newly synthesized cDNA. The subsequent in vitro transcription used T7 RNA polymerase, which simultaneously amplified the target material and incorporated cyanine 3-labeled CTP. cDNA synthesis and in vitro transcription were performed at $40{ }^{\circ} \mathrm{C}$ for $2 \mathrm{~h}$, respectively. The labeled cRNA was purified using the Qiagen RNeasy Mini Kit and quantified on a NanoDrop1000. Finally, $1650 \mathrm{ng}$ cRNA of each sample was hybridized at $65^{\circ} \mathrm{C}$ for $17 \mathrm{~h}$ and the arrays subsequently scanned on an Agilent C-type Microarray Scanner G2565CA.

\section{Microarray data analysis}

The microarray raw data were processed using the Agilent Feature Extraction Software (v10.7). A quantile between array normalization was applied, and differential expression was determined using the Bayesian adjusted $t$-statistic linear model of the "Linear Models for Microarray Data" (LIMMA) [21] package in $\mathrm{R}$. The $p$ values were corrected for a false discovery rate (Benjamini and Hochberg 1995) of $5 \%$, and the gene expression levels were presented as $\log 2$-transformed intensity values. 
Normalized gene expression data have been deposited in NCBI's Gene Expression Omnibus (GEO) and are accessible through GEO Series accession number GSE143408. Probes significantly different between the two groups were identified with an adjusted $p$ value of $\leq$ 0.05 and an average absolute fold change of $\geq 1.5$. For functional annotation and gene network analysis, filtered gene lists were examined using QIAGEN's Ingenuity Pathway Analysis (IPA ${ }^{\circ}$, QIAGEN, Redwood City, www. qiagen.com/ingenuity) and "Gene Set Variation Analysis" (GSVA) [22], "Gene Set Enrichment Analysis" (GSEA) [23], "Gene Ontology enRIchment anaLysis and visuaLizAtion tool" (GOrilla) [24], and GOsummaries [25].

\section{Comparative gene signature scoring}

Gene sets of indicated signatures were acquired from Kyoto Encyclopedia of Genes and Genomes (KEGG), Gene Ontology, Ingenuity Pathway Analysis, REACTOME, and the Molecular Signature Database (hallmark gene sets, Broad Institute). GEO-deposited RNAseq data sets GSE104935 [26], GSE88752 [27], and GSE48403 [28] were downloaded as raw counts and processed by an edgeR pipeline with TMM normalization to obtain fragments per kilobase of transcript (fpkm) values. Mean expression was used to collapse multiple isoforms. Microarray data of this study were processed through limma pipeline, and Ensembl v77 probes were collapsed to gene level using mean $\log 2$ intensities. GSVA [22] was used for signature scoring, and non-scaled bubble plots were created with Morpheus webtool, with color indicating the direction of change of the GSVA scores (red $=$ increased scores/gene sets increase in overall expression, blue $=$ decreased scores/gene sets decrease in overall expression).

\section{Quantitative single cell analysis ( $\mathrm{qSCl}$ ) of lipid content by fluorescent microscopy}

Prior to seeding, 96-well Ibidi optical plates were coated with $150 \mu \mathrm{l}$ poly-L-ornithine (PLO, Sigma) and washed with PBS to increase cell attachment. PCa cell lines pretreated with either 5\% FBS + enzalutamide $(10 \mu \mathrm{M})$ or $5 \%$ CSS were harvested three days prior completion of the indicated treatment times by trypsinization and seeded into PLO-coated 96-well Ibidi optical plates at a density of 6000 cells/well in their corresponding types of media (RPMI medium supplemented with either 5\% FBS (D0), $5 \% \mathrm{FBS}+$ enzalutamide $(10 \mu \mathrm{M})$, or $5 \%$ CSS $)$. After 3 days, the growth medium was removed, and the cells were washed with PBS and fixed with $4 \%$ paraformaldehyde (PFA). Lipids were stained with $0.25 \mu \mathrm{g} / \mathrm{ml}$ Nile Red (Sigma) overnight as described previously [29], and nuclear DNA was counterstained with $1 \mu \mathrm{g} / \mathrm{ml}$ 4',6-diamidino-2phenylindole (DAPI, Thermo Fisher). Alternatively, free cholesterol was stained with $50 \mu \mathrm{g} / \mathrm{ml}$ Filipin (Sigma), and DNA was counterstained with Draq5 $(1 \mu \mathrm{M}$, Thermo Fisher). 500 cells/well were imaged using an InCell 2200 automated fluorescence microscope system (GE Healthcare Life Sciences). Quantitative analysis was performed with Cell Profiler Software [30] to measure total cellular content of neutral lipids, phospholipids, and free cholesterol based on mean fluorescence intensity and morphometric analysis of lipid droplets.

\section{qSCl of lipid transporter expression by immunofluorescence microscopy}

Seventy-two hours after seeding of ATT pre-treated LNCaP cells in PLO-coated 96-well Ibidi optical plates and PFA fixation as described above, cells were permeabilized with TBS-0.1\% Triton X-100 in PBS for $5 \mathrm{~min}$ followed by a $10 \mathrm{~min}$ treatment with TBS-2\% BSA to block non-specific binding. Primary antibodies (LDLR (Abcam, ab52818) and SCARB1 (Abcam, ab217318)) were added at a 1:100 dilution in TBS-2\% BSA $(60 \mu \mathrm{l} /$ well). After $24 \mathrm{~h}$, the primary antibody was removed and cells were washed with TBS-0.1\% Triton X-100 3 times for $5 \mathrm{~min}$ each. Secondary antibody (Alexa Fluor 568, Thermo Fisher) was added to cells at a 1:250 dilution in TBS-2\% BSA ( $60 \mu \mathrm{l} /$ well). After $1 \mathrm{~h}$ in the dark, the secondary antibody was removed and cells were washed with TBS-0.1\% Triton X-100 3 times for 5 min each. DNA and F-actin were counterstained for $20 \mathrm{~min}$ in the dark with $1 \mu \mathrm{l} / \mathrm{ml} \mathrm{4',6-diamidino-2-phenylindole} \mathrm{(DAPI,}$ Invitrogen) and Alexa Fluor 647 phalloidin (Thermo Fisher), respectively. Staining solution was replaced with $200 \mu \mathrm{l}$ PBS. Imaging and quantitative analysis were carried out as described above using the InCell 2200 System and CellProfiler software.

\section{$\mathrm{qSCl}$ of metabolic processes}

ATT pre-treated cells were seeded as described above. For quantifying C16:0 fatty acid uptake, growth media was exchanged with $65 \mu \mathrm{l} /$ well of serum-free RPMI media (Thermo Fisher) supplemented with $0.2 \%$ BSA (lipid-free, Sigma) and C16:0-Bodipy $(5 \mu \mathrm{M}$, Thermo Fisher) and Mitotracker Orange CMTMRos $(0.4 \mu \mathrm{M}$, Thermo Fisher) and incubated at $37^{\circ} \mathrm{C}$ for $1 \mathrm{~h}$. For quantifying cholesterol uptake, the growth medium was exchanged with $65 \mu \mathrm{l} /$ well of serum-free RPMI media $(0.2 \%$ lipid-free BSA) supplemented with $15 \mu \mathrm{M}$ NBD (22-(N(7-Nitrobenz-2-Oxa-1,3-Diazol-4-yl) Amino-23,24-Bisnor-5-Cholen-3 $\beta-O l$, Thermo Fisher) and incubated at $37^{\circ} \mathrm{C}$ for $2 \mathrm{~h}$. For quantifying lipoprotein complex uptake, the growth medium was exchanged with $65 \mu \mathrm{l} /$ well of serum-free RPMI media (0.2\% lipid-free BSA) supplemented with 1,1'-Dioctadecyl-3,3,3',3'-Tetramethylindocarbocyanine (DiI)-labeled acetylated-LDL $(15 \mu \mathrm{g} / \mathrm{ml}$, Thermo Fischer) or DiI-labeled LDL $(15 \mu \mathrm{g} / \mathrm{ml}$, Thermo 
Fisher) and incubated at $37^{\circ} \mathrm{C}$ for $2 \mathrm{~h}$. Phosphatidylethanolamine uptake was measured as described above in serum-free RPMI media ( $0.2 \%$ lipid-free BSA) or, where noted, in conditioned media by incubation of cells at $37^{\circ} \mathrm{C}$ for $1 \mathrm{~h}$ with NBD-PE $(5 \mu \mathrm{M}, 22-(\mathrm{N}-$-(7-Nitrobenz-2-Oxa-1, 3-Diazol-4-yl)-1, 2-Dihexadecanoyl-sn-Glycero-3-Phosphatidylethanolamine) triethylammonium salt, Thermo Fisher). After incubation, cells were fixed with 4\% PFA. Cellular DNA and F-actin were then counterstained with DAPI and Alexa Fluor 647 Phalloidin (Thermo Fisher). Image acquisition and quantitative analysis were performed as described above.

For measuring cellular uptake of lyso-PC-A2, cells were seeded and processed as described above and incubated for $60 \mathrm{~min}$ at $37^{\circ} \mathrm{C}$ with $65 \mu \mathrm{l} /$ well of conditioned media supplemented with $5 \mu \mathrm{M}$ Red/Green BODIPY ${ }^{\circ}$ PC-A2 (A10072, 1-O-(6-BODIPY ${ }^{ø}$ 558/568-aminohexyl)-2-BODIPY ${ }^{\circ}$ FL C5-sn-glycero-3-phosphocholine, Thermo Fisher). This phospholipid derivative carries in close proximity a red and green Bodipy fluorophore on the sn-1 and sn-2 acyl chains respectively that provides a capacity for dual emission fluorescence ratio detection. Cleavage of the BODIPY $^{\circ}$ FL pentanoic acid substituent at the sn- 2 position by type 2 phospholipases results in decreased quenching by fluorescence resonance energy transfer (FRET) to the BODIPY ${ }^{\circ} 558 / 568$ dye attached to the sn- 1 position. The result is a lipase-dependent increase in BODIPY $^{\circ}$ FL fluorescence emission detected in the range 515-545 nm (green). The FRET-sensitized BODIPY ${ }^{\circ}$ 558/ 568 fluorescence signal is expected to show a reciprocal decrease. Notably, dose titration experiments with unconjugated BODIPY ${ }^{\ominus}$ FL pentanoic acid (Thermo Fisher) suggested a passive and slow route of cellular uptake when compared to longer chain FAs (C12:0 and C16:0, data not shown). Following the incubation, cells were fixed with $4 \%$ PFA, and cellular DNA and F-actin were then counterstained with DAPI and Alexa Fluor 647 Phalloidin (Thermo Fisher). Image acquisition and quantitative analysis were performed as described above. Lyso-PC-A2 uptake was calculated as ratio of green (cleaved lyso-PC-A2) vs red (uncleaved PC-A2) signal.

Membrane fluidity (differences in membrane lipid packaging) was measured by live ratio-fluoresce microscopy of ATT pre-treated cells after incubation in $80 \mu \mathrm{l} /$ well of DPBS supplemented with $1 \mu \mathrm{M}$ di-4ANEPPDHQ (D36802, Thermo Fisher) for $30 \mathrm{~min}$ at $37^{\circ} \mathrm{C}$. DNA was counterstained with Hoechst 33342 $(1 \mu \mathrm{g} / \mathrm{ml}$, Sigma-Aldrich), and cells were washed once with DPBS. di-4-ANEPPDHQ-labeled cells $(n>750$ cells, 8 wells/sample, 2 biological replicates) were imaged at $560 \mathrm{~nm}$ and $650 \mathrm{~nm}$ using the InCell 2200 System, where a spectral red shift is indicative of an increase in the disordered/liquid phase of the plasma membrane. Quantitative image analysis and calculation of the $560 \mathrm{~nm} / 650 \mathrm{~nm}$ ratio was carried out with CellProfiler software.

Lipid peroxidation was measured by live ratiometric fluorescence microscopy of ATT pre-treated cells after incubation in $65 \mu \mathrm{l} /$ well of serum-free RPMI media (0.2\% lipid-free BSA) supplemented with $5 \mu \mathrm{M}$ with BODIPY 581/591 C11 (Thermo Fisher) for $60 \mathrm{~min}$ at $37^{\circ} \mathrm{C}$. Peroxidation of the polyunsaturated butadienyl portion of the fluorescent dye results in a spectral fluorescence emission shift from orange $(\sim 590 \mathrm{~nm})$ to green $(\sim 510 \mathrm{~nm})$.

\section{Measurement of cell confluence and $\mathrm{qSCl}$ of cell death}

Cell proliferation as a function of increasing cell confluence was measured by live imaging microscopy with the IncuCyte FLR and Zoom system (Essen BioScience, Ann Arbor, MI, USA). Parental or ATT pre-treated PCa cells were seeded as described above in 96-well Essen ImageLockTM plates (Essen BioScience, Ann Arbor, MI, USA). Images were acquired at 2-h intervals with $\mathrm{a} \times 10$ objective for up to 7 days.

For assessment of cell death, cells were seeded in 96 well plates $(n=3$ wells/treatment with $>4000$ cells/ wells) as described above and treated with indicated compounds. At the end of the treatment period, cells were co-stained with Hoechst $3342(1 \mu \mathrm{g} / \mathrm{ml}$, total cell count) and propidium iodide $(5 \mu \mathrm{g} / \mathrm{ml}$, Sigma-Aldrich, dead cells). Cells were imaged using the InCell 2200 System, the images were analyzed with Cell Profiler software (Broad Institute) for total and dead cell count, and the percentage of dead cells was calculated based on the ratio of dead and total cells.

\section{Lipidome analysis}

All extractions were performed in $2 \mathrm{ml}$ glass vials. Methanol $(220 \mu \mathrm{L})$ was added to cell pellet of approximately 2 million cells and vortexed. Internal standard (40 $\mu$ S SPLASH Lipid-o-mix deuterated internal standard obtained from Avanti Polar Lipids, Alabaster, AL, and $20 \mu \mathrm{l}$ n-Nonadecanoic acid (C19:0)) was added and briefly vortexed. Seven hundred seventy microliters of MTBE was added, and the mixture was incubated for 1 $\mathrm{h}$ at room temperature in a shaker. Phase separation was induced by adding $200 \mu \mathrm{l} \mathrm{NH}_{4} \mathrm{CH}_{3} \mathrm{CO}_{2}(150 \mathrm{mM})$. After vortexing for $20 \mathrm{~s}$, the sample was centrifuged at 20,000g for $5 \mathrm{~min}$. The upper (organic) phase was collected and stored at $-80^{\circ} \mathrm{C}$, then diluted into $2: 1 \mathrm{MeOH}$ to $\mathrm{CHCl}_{3}$ with $7.5 \mathrm{mM} \quad \mathrm{NH}_{4} \mathrm{CH}_{3} \mathrm{CO}_{2}$ for mass spectrometry analysis.

Tandem mass spectrometry (MS) of the intact lipids was performed using a triple quadrupole mass spectrometer (6500 QTRAP, SCIEX, ON, Canada). The lipid extracts (as described above) were diluted 40-fold prior to analysis. Samples were directly infused into the 
electrospray ionization source of the mass spectrometer using a loop injection method, where $100 \mu \mathrm{l}$ of sample was loaded into a sample loop using an autosampler and subsequently infused into the mass spectrometer by electrospray ionization at a flow rate of $20 \mu \mathrm{l} / \mathrm{min}$. Lipid classes were targeted using either precursor ion or neutral loss scans. For quantification, SPLASH was added to cells prior to lipid extraction. Tandem MS data was processed using LipidView (version 1.3beta; SCIEX) using predefined target lists.

Hydrolysis and derivatization of lipids extracts to fatty acid methyl esters (FAME) was performed on-line using trimethylsulfonium hydroxide [31]. FAMEs were analyzed with a gas chromatograph coupled to a mass spectrometer (GC/MS - TQ8040; Shimadzu, Kyoto, Japan). The separation was carried out on a RTX-2330 capillary column (cyanopropyl stationary phase, $60 \mathrm{~m} \times 0.25 \mathrm{~mm}$, film thickness $0.20 \mu \mathrm{M}$; Restek, Bellefonte, PA, USA), and the electron ionization energy was set at $70 \mathrm{eV}$. Conditions for the analysis of FAMEs were as follows: carrier gas, He: column flow at $1 \mathrm{ml} / \mathrm{min} ; 22: 1$ split ratio, injection volume $1 \mu \mathrm{l}$; injector temperature $240{ }^{\circ} \mathrm{C}$; interface and ion source temperature $260 \mathrm{C}$. GC oven temperature was maintained at $100{ }^{\circ} \mathrm{C}$ for $1 \mathrm{~min}$, thermal gradient 100 to $140{ }^{\circ} \mathrm{C}$ at $10{ }^{\circ} \mathrm{C} / \mathrm{min}, 140$ to $175{ }^{\circ} \mathrm{C}$ at 6 ${ }^{\circ} \mathrm{C} / \mathrm{min}$, and 175 to $200{ }^{\circ} \mathrm{C}$ at $10{ }^{\circ} \mathrm{C} / \mathrm{min}$ and hold for 1 min, followed by 200 to $250{ }^{\circ} \mathrm{C}$ at $5{ }^{\circ} \mathrm{C} / \mathrm{min}$ and hold for $4 \mathrm{~min}$. The data were acquired with Q3 scan mode from $m / z 50-650$. For data collection, the MS spectra were recorded from 4.6 to $28.33 \mathrm{~min}$. The data was processed in GC/MS solution software (Shimadzy, Kyoto, Japan). Fatty acids were identified based on retention time alignment with reference FAMEs from a mixture of standards (Food Industry FAME mix (Table S6), Restek, Bellefonte, PA, USA). All samples were normalized to internal standard C19:0, and peak areas of relevant FAMEs were subtracted from the negative control samples.

\section{${ }^{13} \mathrm{C}$ carbon tracing of metabolites by mass spectrometry} LNCaP cells were treated with DMSO or enzalutamide $(10 \mu \mathrm{M})$ for up to 21 days as described above. Seventytwo hours prior to harvesting, media was replaced with complete growth media supplemented with uniformly labeled ${ }^{13} \mathrm{C}$-acetate $(500 \mu \mathrm{M}$, Sigma-Aldrich). Metabolites were extracted using $80 \%$ methanol in water extraction buffer (Sigma) that was supplemented with uniformly deuterated myristic acid ( $2 \mu \mathrm{M}$, Sigma) as an internal control. Samples were normalized based on total protein measurements in precipitates by bicinchoninic acid assay (BCA, Pierce).

GC/MS analyses were performed using an Agilent $7890 \mathrm{~A}$ GC equipped with a DB-35MS $(30 \mathrm{~m}, 0.25 \mathrm{~mm}$ i.d., $0.25 \mu \mathrm{m}$ ) capillary column (Agilent Technologies), interfaced with a triple quadruple tandem mass spectrometer (Agilent 7000B, Agilent Technologies) operating under ionization by electron impact at $70 \mathrm{eV}$. The injection port, interface, and ion source temperatures were maintained at $230{ }^{\circ} \mathrm{C}$. The temperature of the quadrupoles was maintained at $150{ }^{\circ} \mathrm{C}$. The injection volume was $1 \mu \mathrm{l}$, and samples were injected at a 1:25 split ratio. Helium flow was kept constant at $1 \mathrm{ml} / \mathrm{min}$.

GC oven temperature was maintained at $60^{\circ} \mathrm{C}$ for 1 min, increased to $300^{\circ} \mathrm{C}$ at $10.0^{\circ} \mathrm{C} / \mathrm{min}$. Post-run temperature was $325^{\circ} \mathrm{C}$, kept for $5 \mathrm{~min}$. The mass spectrometer operated in SIM mode and cholesterol-tms derivative was determined from the fragment at $\mathrm{m} / \mathrm{z} 458,4$ (C30H54OSi) as $\mathrm{M}+0$; also, $\mathrm{m} / \mathrm{z}$ fragments representing $M+1$ up to $M+16$ were detected in order to determine the ${ }^{13} \mathrm{C}$-acetate incorporation into cholesterol.

\section{Statistical analysis}

Statistical analysis was performed with GraphPad Prism 8.3 (GraphPad Software, San Diego, CA) and R Studio (RStudio, Boston, MA). Data reported and statistical tests are described in the figure legends.

\section{Results}

Therapy-induced remodeling of metabolic networks leads to cellular quiescence and multidrug tolerance

Our previous longitudinal analysis of LNCaP tumor xenografts in a model of CRPC progression following castration showed that CRPC tumors contained significantly higher lipid levels than tumors resected from sham-castrated mice [32]. These lipids included essential fatty acid (FA) derivatives, indicating that development of CRPC is associated with enhanced uptake of exogenous, diet-derived lipids [16]. A detailed review of transcriptomics data from this study by gene set variation analysis (GSVA) revealed that castration caused a negative enrichment of pathways involved in lipogenesis, mitochondrial activity, and oxidative phosphorylation in tumors at nadir of serum PSA levels compared to noncastrated tumor-bearing mice (intact, control). In support of therapy-induced changes to tumor lipid supply, tumors at PSA nadir showed increased enrichment in pathways of lipoprotein metabolism, lipid storage, lipid transporter, and lysosomal activity (Fig. 1). The complete longitudinal analysis including that from regressing and recurrent tumor samples is shown in Fig. S1A, highlighting the dynamic changes in response to androgentargeted therapies (ATTs) during progression to CRPC.

To investigate therapy-induced longitudinal changes to lipid metabolism on the functional and molecular level, the AR-positive, androgen-dependent LNCaP PCa cell line was grown for up to 21 days in the presence of enzalutamide (Enz) to block AR function. Pairwise sequential comparisons of transcriptome data generated from samples taken prior (day 0 ) and 7,14 , and 21 days post 


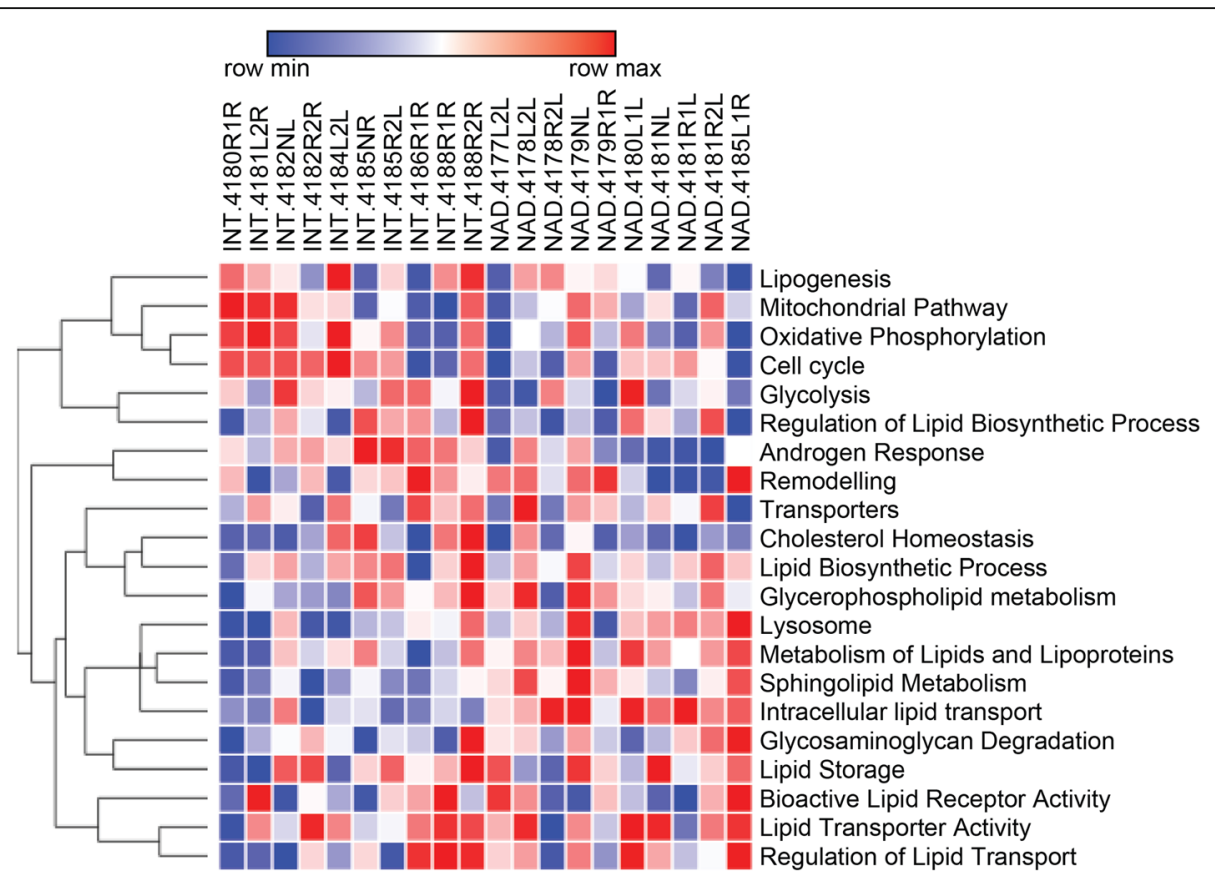

Fig. 1 Therapy-induced reprograming of metabolic networks in a longitudinal PCa tumor xenograft model of CRPC progression. a Gene set variation analysis (GSVA) of ten tumors at PSA nadir (NAD) compared to ten tumors from sham-castrated mice (INT, intact) was generated based on previously published microarray analysis of LNCaP xenograft tumors resected at different time points after castration [32]. Heatmaps were generated with a hierarchical clustering algorithm using completed linkage and Euclidean distance measures and scaled by $z$ score (red $=$ positive $z$ score, blue $=$ negative $z$ score)

commencement of Enz treatment showed that LNCaP cells reached transcriptional stasis after 14 days of Enz, i.e., the set of 4524 differentially expressed genes (D0 vs D14) remained unchanged when compared to 21 days Enz (D0 vs D21, Fig. 2a). This suggested that the phenotype induced by Enz was transcriptionally established by day 14 of Enz treatment and persisted for another week. This longitudinal analysis further highlighted that Enz treatment periods beyond prevailing protocols to assess the acute response to ATTs in vitro (2-4 days) [16] identified an additional 908 (25\%) differentially expressed genes.

GSVA yielded enrichment scores that clustered pathways into three types of adaptive responses to Enz treatment when compared to control (day 0): pathways that changed to and persisted as either negatively or positively enriched and dynamic pathways that, after temporarily displaying negative enrichment (days 7 and 14), reverted to a positive enrichment seen prior treatment commencement. GSVA revealed negative enrichment for known androgen-activated pathways, e.g., lipogenesis, cell cycle, and mitochondrial pathways, including oxidative phosphorylation (Fig. 2b). Pathways with positive enrichment were associated with lysosomal function, lipid metabolism, lipid remodeling, and extra- and intracellular lipid transport (Fig 2b). Dynamic pathways with transient negative enrichment that reverted to their initial positive enrichment seen prior the start of treatment were associated with the androgen response, lipid transporters, cholesterol homeostasis, glycolysis, lipid storage, and regulation of lipid biosynthesis and lipid transport (Fig. 2b). GSVA suggested a substantial reconfiguration of the lipid metabolism network in response to Enz over the treatment period of 21 days and were consistent with the in vivo results (Fig. 1). Consistent with this, protein mass spectrometry of LNCaP cells treated for 0 and 21 days with Enz and gene ontology analysis revealed enrichment of membrane lipid metabolic processes, glycolipid metabolism, and lysosome function (Fig. S1B and Table S2).

Extended cell culture (several months) in androgendepleted or AR-antagonized conditions ultimately yielded androgen-independent or Enz-resistant CRPC cells that reinitiated proliferation $[6,8,33,34]$. Our comparative signature scoring of transcriptomics data sets of relevant studies performed in cell culture leading to Enz resistance in LNCaP and C4-2B cells [26], in castrated mice progressing to primary and secondary CRPC after serial tumor xenograft transplantation of LAPC9 and LNCaP cells [27], and matching PCa tumors from patients before and after androgen deprivation therapy for 22 weeks [28] by GSVA using lipid metabolismrelated gene sets showed extensive concordance between cell culture models, pre-clinical tumor xenograft models, 
A

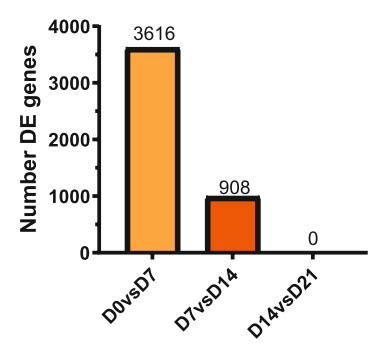

B

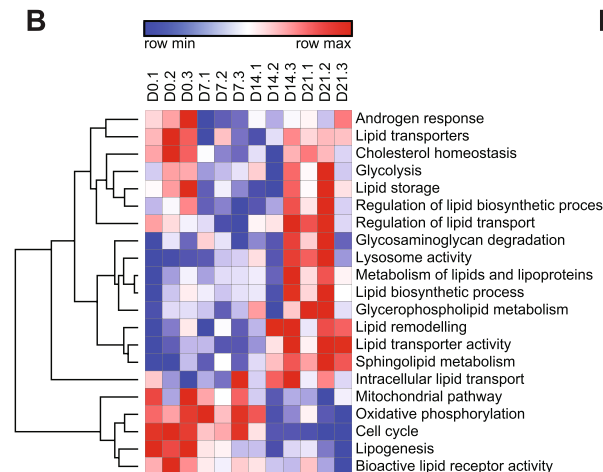

C

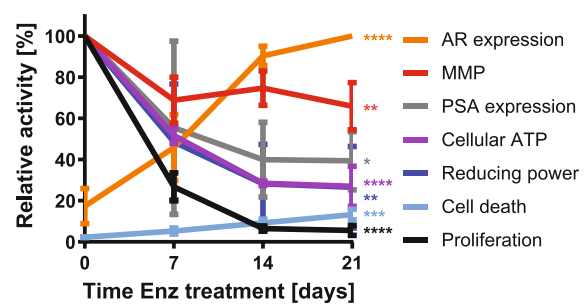

D

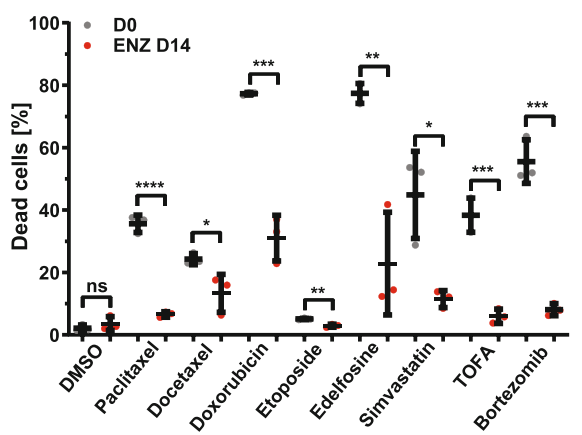

Fig. 2 Therapy-induced metabolic reprogramming of $L N C a P$ PCa cells is associated with cellular quiescence and multi drug tolerance. a $L N C a P$ cells were treated for $0,7,14$, and 21 days with AR antagonist enzalutamide (Enz, $10 \mu \mathrm{M})$, and samples were analyzed by microarray analysis. The number of differentially expressed genes between indicated pairwise comparisons are shown (FDR 1.5fold, $p<0.05$ ). $\mathbf{b}$ Gene set enrichment analysis of the microarray data described in a for the analysis of indicated pathways. $\mathbf{c} L N C a P$ cells were treated as in $\mathbf{a}$, and samples were analyzed by qRT-PCR (AR and PSA mRNA expression), live-cell confluence imaging (proliferation), CellTiter-Glo assay (cellular ATP levels), PrestoBlue assay (cellular reducing power), quantitative single-cell imaging (qSCl) of MitoTracker Orange CMTMRos (mitochondrial membrane potential, MMP), and dead/live staining (cell death) for functional characterization of Enz-induced metabolic changes ( $n=3$, activities relative to day 0 with the exception of AR levels, where levels were calculated relative to day 21). $\mathbf{d}$ LNCaP cells were cultured in growth media without (D0)

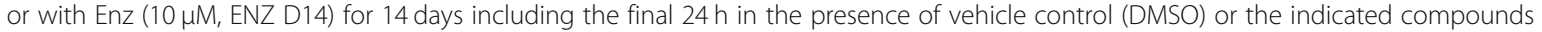
(paclitaxel $2.5 \mu \mathrm{M}$, docetaxel $2.5 \mu \mathrm{M}$, doxorubicin $2.5 \mu \mathrm{M}$, etoposide $5 \mu \mathrm{M}$, edelfosine $7.5 \mu \mathrm{M}$, simvastatin $30 \mu \mathrm{M}$, TOFA $60 \mu \mathrm{M}$, bortezomib $2.5 \mu \mathrm{M}$ ). The percentage of dead cells was calculated based on qSCl of Hoechst 3342 (total cell count) and propidium iodide (dead cells) staining ( $n=3$ wells/treatment with $>4000$ cells/well; ns, not significant, ${ }^{*} p<0.05$, ${ }^{* *} p<0.01$, ${ }^{* * *} p<0.001$, ${ }^{* * * *} p<0.0001$, one-way ANOVA followed by Dunnett's multiple comparisons test compared to D0, representative result of 2 independent experiments)

and clinical samples of ATT resistance (Fig. S1C). This analysis further highlighted the dynamic nature of some therapy-induced metabolic changes, potentially limiting their value as co-treatment options based on the therapeutic window.

The observed time-dependent increase in AR mRNA expression by Enz over 21 days (Fig 2c, Fig. S2A) was consistent with reported resistance mechanisms to ATT $[2,4]$. mRNA levels of PSA/KLK3 remained decreased (Fig. 2c) and a large subset of other AR-activated and suppressed genes (Fig. S2B) did not change, suggesting that AR activity remained suppressed. Consistent with the de-differentiation effect of AR-antagonism, transcriptional levels of several stemness markers were significantly increased (Table S1) [35]. Furthermore, Enztreated LNCaP cells showed a time-dependent strong reduction in cellular reducing power $(-70 \%)$, ATP levels $(-60 \%)$, and mitochondrial membrane potential $(-30 \%)$, which stabilized after 14 days (Fig. 2c, Fig. S2C). Proliferation almost completely ceased after 14 days of Enz treatment, but cell death was only modestly increased (Fig. 2c, Fig. S2D) which was possibly due to protection via significantly increased autophagy (Fig. S2E). This low cycling phenotype was underpinned by a strong downregulation of genes associated with cell cycle progression (Fig. 2b). Enz-treated LNCaP cells adopted a spindle-like cell morphology marked by a reduced cell size, increased cell perimeter, and major axis length (Fig. S2F). Transcriptionally, metabolically, and proliferation-wise, LNCaP cells responded to extended Enz treatment by entering into a state of cellular quiescence. Associated with this phenotype was the acquisition of multi-drug tolerance (MDT) to a range of lipogenesis inhibitors and clinically relevant chemotherapeutics for CRPC in LNCaP, C4-2B, and DuCaP cells (Fig. 2d and S2G), indicating that this adaptive response to Enz is shared across multiple AR-positive PCa cell lines. Despite MDT, a limited drug screen revealed enhanced sensitivity of $\mathrm{PCa}$ cells in the Enz-tolerant state to U18666A (Fig. S2H), an inhibitor of the lysosomal Niemann-Pick C1 protein 
(NPC1). NPC1 together with NPC2 are critical for lysosome to cytoplasm transport of exogenous cholesterol [36], and both proteins show strongly increased expression in localized and metastatic PCa (Fig. S2H). Notably, culturing of LNCaP cells in androgen-depleted growth media (CSS) for 14 days showed similar adaptive responses when compared to Enz, e.g., cellular ATP levels and reducing power, proliferation, and MDT (Figs. S2C, S2D, and S2G), suggesting that both ATTs cause similar metabolic reprograming that leads to the persister cell state.

\section{Androgen-targeted therapy increased cellular lipid content}

The above results suggested major changes to lipid metabolism networks by Enz. Previous studies, including our own work, have demonstrated that androgens strongly enhance cellular lipid content of $\mathrm{PCa}$ cells through enhanced lipogenesis $[37,38]$ and lipid uptake [16] and that AR antagonists block these lipid supply pathways. To measure Enz-induced changes to the cellular content of neutral lipids, lipid droplets, phospholipids, and free cholesterol, we used quantitative singlecell imaging (qSCI) of two fluorescent lipophilic dyes, Nile Red and Filipin. Nile Red is able to distinguish between phospholipids and neutral lipids [e.g., triacylglycerols (TAGs) and cholesteryl esters (CEs) stored in lipid droplets] based on their difference in hydrophobicity having separate fluorescence emission maxima [39]. Filipin is a selective fluorescent stain for free, non-esterified cholesterol which is mainly found in the plasma membrane [40]. Contrary to the expected lipid depletion resulting from loss of androgen-enhanced lipid supply, qSCI of Nile Red-stained LNCaP cells demonstrated that Enz significantly increased neutral lipid and phospholipid content with increasing time of treatment (Fig. 3a-c). Morphometric analysis of lipid droplets (LDs) showed that the LD number and average total area of LDs per cell was also significantly increased with treatment time (Fig. 3b top and middle panel). Consistent with this, the mRNA expression of the LD surface protein PLIN1 was significantly increased by Enz treatment (Fig. 3b bottom panel). qSCI of Filipin-stained LNCaP cells showed that Enz induced a significant increase in free cholesterol content (Fig. 3d). Similar observations were made when LNCaP cells were maintained in androgen-depleted media (CSS, data not shown).

For molecular analysis of the Enz-induced longitudinal changes to the lipidome, we next ran electrospray ionization mass spectrometry (ESI-MS) of lipidomics of lipid extracts prepared from LNCaP cells treated with vehicle control (FBS) or for 7, 14, and 21 days with Enz. Principal component analysis showed that each time point sample group of the Enz-treatments clustered distinctly from the vehicle treated control group (FBS) and to a lesser extent from each other (Fig. S3A). LNCaP cells significantly increased cellular levels of sphingomyelin (SM), phosphatidylcholine (PC), phosphatidylethanolamine (PE), phosphatidylserine (PS), and phosphatidylglycerol (PG) over the time course of treatment (Fig. 3e left panel, Fig. S3B-F). While all major membrane lipid classes were increased by Enz, neutral lipids like CEs and TAGs stored within LDs were significantly decreased (Fig. 3e right panel, Figs. S3G and S3H). Of the top 50 deregulated lipid species measured, $86 \%$ of these were found to have increased abundance with increasing time of Enz treatment (Fig. S3I). Notably, the majority of these were PC and PE phospholipids that make up 70\% of the lipid content of mammalian cells [41], providing further evidence that Enz increased cellular lipid content. Together, qSCI and lipidomics analyses demonstrated that Enz induced substantial remodeling of all major lipid classes, leading to a net increase in cellular lipid content.

\section{Enhanced lipid uptake fuels cellular lipid accumulation}

As shown above, therapy-induced persister cells displayed multidrug tolerance to lipogenesis inhibitors (Fig. $2 \mathrm{~d}$ ), suggesting that de novo synthesis played a limited role in cellular lipid accumulation. Indeed, metabolic tracing of ${ }^{13} \mathrm{C}$-acetate by mass spectrometry revealed that de novo cholesterol synthesis decreased by $71 \%(p>$ 0.0001) after Enz treatment of LNCaP cells for 21 days when compared to control (Fig. 4a). No significant incorporation of ${ }^{13} \mathrm{C}$-glucose, another key carbon source for lipogenesis, into lipids was measured (data not shown), and already low basal glucose uptake was significantly reduced by Enz (Fig. S4A). Consistent with this, transcriptomics and proteomics analysis showed that key enzymes of de novo cholesterol and FA synthesis pathways were significantly downregulated by Enz treatment (Fig. 4b, Table S1), suggesting that alternative lipid supply pathways were responsible for therapyinduced lipid accumulation.

To measure cellular lipid uptake, qSCI with various fluorophore labeled lipid probes (NBD-cholesterol, DiILDL, Dil-acetylated LDL, Bodipy-C16:0, NBDphosphatidylethanolamine, Bodipy-phosphatidylcholine) was conducted. Enz treatment significantly increased cellular uptake of free NBD-cholesterol and DiI-complexed low-density lipoprotein (LDL) and acetylated LDL in LNCaP cells, as well as NBD-phosphatidylethanolamine and Bodipy-lyso-phosphatidylcholine in LNCaP and C42B cells (Fig. 4c, d) in a time-dependent manner. Consistent with increased lipid uptake and cellular content, Enz significantly increased the mRNA levels of several lipid transporters, including phospholipid transporters and cofactors belonging to the family of P4-ATPases/flippases (Fig. 4e, top panel; Table S1) and other cargo-selective 


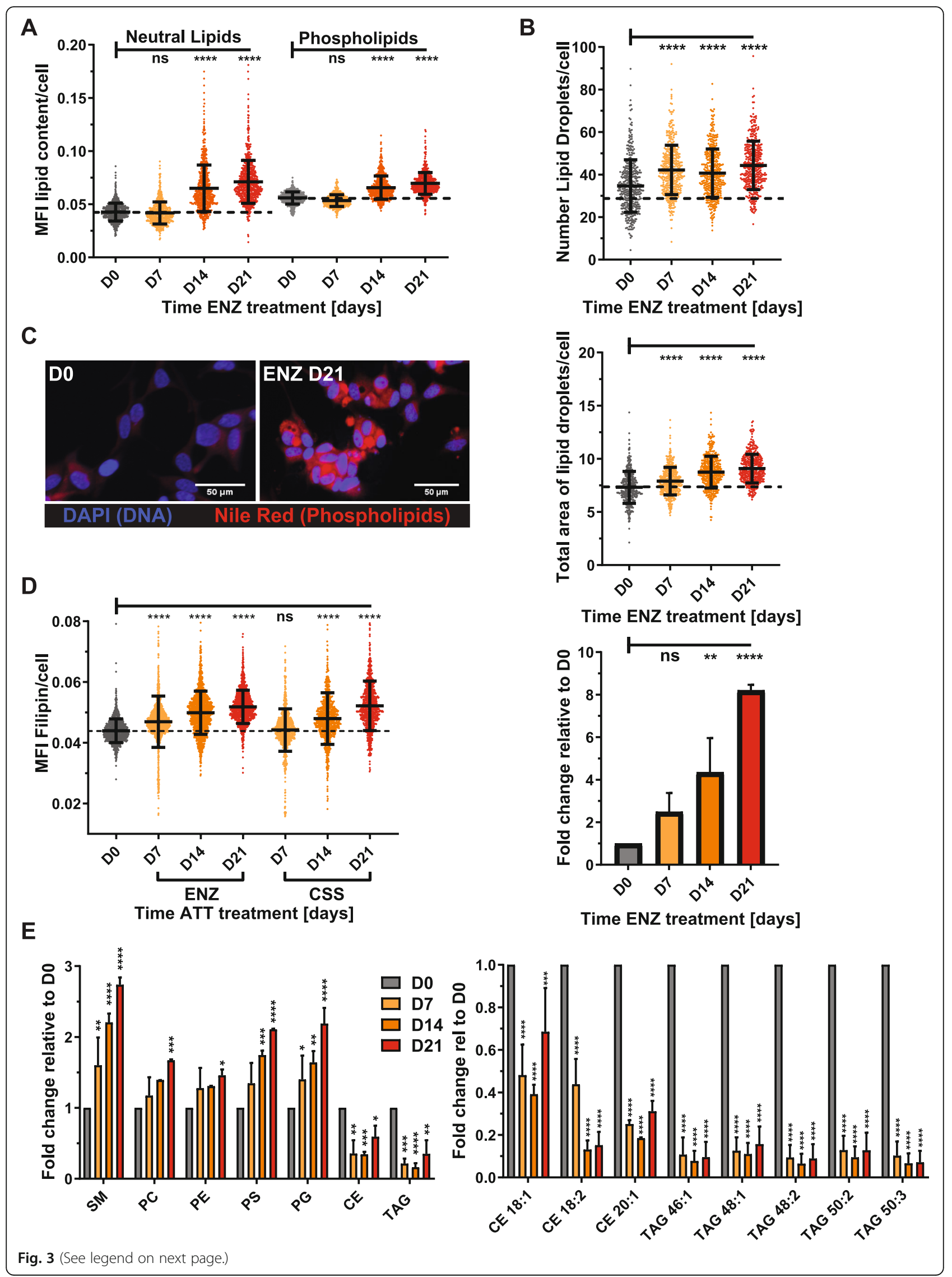


(See figure on previous page.)

Fig. 3 Enzalutamide increased cellular lipid content. LNCaP cells were treated for the indicated times with Enz (10 $\mu \mathrm{M})$ and analyzed by qSCl based on the mean fluorescence intensity (MFI) of Nile Red to measure $\mathbf{a}$ cellular neutral lipid and phospholipid content and $\mathbf{b}$ number of lipid droplets (LDs)/cell and total area of LDs/cell $(n>9000$ cells/treatment, mean \pm SD, representative result of 3 independent experiments). Perilipin mRNA expression was measured by qRT-PCR $(n=3$, mean \pm SD). c Representative images of D0 and D21 showing phospholipid and DNA staining as described in a. $\mathbf{d}$ LNCaP cells were treated as in $\mathbf{a}$ or grown in androgen-depleted media (CSS, charcoal-stripped serum) for the indicated times, stained with Filipin, and free cholesterol was measured by qSCI $(n>9000$ cells/treatment, mean \pm SD, representative result from 2 independent experiments). e LC/MS shotgun lipidomics of intact lipid species of LNCaP cells treated as described in $\mathbf{a}$. Total combined sum compositions of indicated lipid classes (left panel, SM, sphingomyelin; PC, phosphatidylcholine; PE, phosphatidylethanolamine; PS, phosphatidylserine; PG,

phosphatidylglycerol; CE, cholesterol ester; TAG, triacylglycerol), and individual CE and TAG species (right panel) are reported as fold change relative to D0 $(n=2$, mean \pm SD). Statistical analysis: a-d one-way ANOVA followed by Dunnett's multiple comparisons test compared to D0; e two-way ANOVA followed by Tukey's multiple comparisons test relative to D0; ns, not significant, ${ }^{*} p<0.05,{ }^{* *} p<0.01,{ }^{* * *} p<0.001,{ }^{* * * *} p<0.0001$

lipid transporters, including low-density lipoprotein receptor (LDLR) (Fig. 4e bottom panel). Protein expression of several of these lipid transporters was upregulated by several orders of magnitude in clinical samples of bone metastatic CRPC when compared to localized, hormone-naïve PCa and normal prostate gland [42] (Fig. S4B), suggesting that enhanced lipid uptake might remain activated throughout the development of ATT resistance and CRPC.

LDL particles are a bulk supply of apolipoproteins and complex lipids, including CEs, TAGs, and phospholipids to cells via receptor-mediated endocytosis of LDLR and scavenger receptor SCARB1 [43, 44]. Consistent with increased cellular uptake of LDL and acetylated LDL, protein expression of lipid transporters LDLR and SCARB1 was significantly enhanced by Enz. (Fig. 4f). Surprisingly, uptake of saturated free FA (Bodipy-C16:0) was drastically decreased by both androgen-depleted serum (CSS) and Enz treatment over time (Fig. 4g), suggesting that ATT-induced changes to transporter-mediated lipid uptake are specific. Gas chromatography-mass spectrometry (GC/MS) analysis of derivatized FAs released upon saponification of complex lipids revealed that saturated FAs (C16:0 and C18:0) and essential PUFAs linoleic acid (C18:2n-6) and its derivative arachidonic acid (C20:4n-6) were significantly increased by Enz with increasing time of treatment (Figs. 4h and S4C). Together, the above results suggested that saturated FA requirements from exogenous sources were preferably met by uptake of complex lipids (e.g., TAGs, CEs, phospholipids) which are also rich sources of PUFAs (Fig. S4D). Despite this observed cargo selectivity, Enz also enhanced cargo non-selective mechanisms involved in lipid scavenging, e.g., macropinocytosis [45] and tunneling nanotubes (TNTs, Figs. S4E and S4F) [46]. Together, these results demonstrated that Enz-treated PCa cells overall enhanced lipid uptake through cargo-selective and non-selective mechanisms.

\section{Therapy-induced lipid remodeling increases membrane fatty acid desaturation and fluidity}

The above results showed that Enz affected all major lipid classes and strongly increased cellular content of phospholipids, suggesting changes to membrane composition and function. Exemplified by lipid species of the phosphatidylethanolamine (PE) phospholipid class, PEs with shorter FA chains (34:2 and 36:2) were significantly decreased, while PEs with longer and more unsaturated FA chains (38:3, 38:4, 38:5, and 38:6) were significantly increased by Enz treatment compared to control (Fig. 5a). Similar trends were seen in SM, PC, PE, PS, and PG lipid classes (Fig. S3B-F), where an enrichment of phospholipids with long-chain PUFAs was observed. Integrated analyses of all dysregulated fatty acid species detected by GC/MS FAME analysis (Fig. S4C) or proteins detected by proteomics (Fig. S1B) with the mRNA expression changes by microarray (Fig. 2) of LNCaP cell treated with vehicle control or Enz for 21 days demonstrated significant enrichment of multiple pathways involved in PUFA metabolism (Fig. S5A), including those of essential PUFAs (e.g., linoleic acid and arachidonic acid).

Consistent with increased PUFA content, Enz-treated LNCaP cells displayed increased membrane fluidity (Fig. 5b). Regarding the mechanisms underlying the increase in desaturation and elongation of PUFAs, we noted a significant reduction of PUFA containing storage lipids (CEs and TAGs; Figs. S3G and S3H). Apart from enzymes that play primarily a role in the elongation of very long FAs ( $>24$ carbon atoms) and the desaturation of sphingolipids and mono-unsaturation of FAs (ELOVL4 [47], DEGS2 and SCD5), mRNA expression of most FA elongases and desaturases was significantly decreased by Enz (Fig. 5c. Table S1). In support of this, a review of microarray data from our LNCaP tumor xenograft model of CRPC progression [32] revealed that the expression of elongases and desaturases was overall reduced in regressing tumors and tumors at PSA nadir when compared to tumors from sham-castrated mice (Fig. S5B and S5C). Notably, this expression pattern reversed and increased in recurring tumors and CRPC when compared to tumors from sham-castrated mice. Furthermore, FA elongation and de novo FA synthesis share the requirement of malonyl-CoA as a $\mathrm{C} 2$ carbon donor, which is produced by acetyl-CoA carboxylase (ACACA). As shown in Fig. 4b, ACACA mRNA 


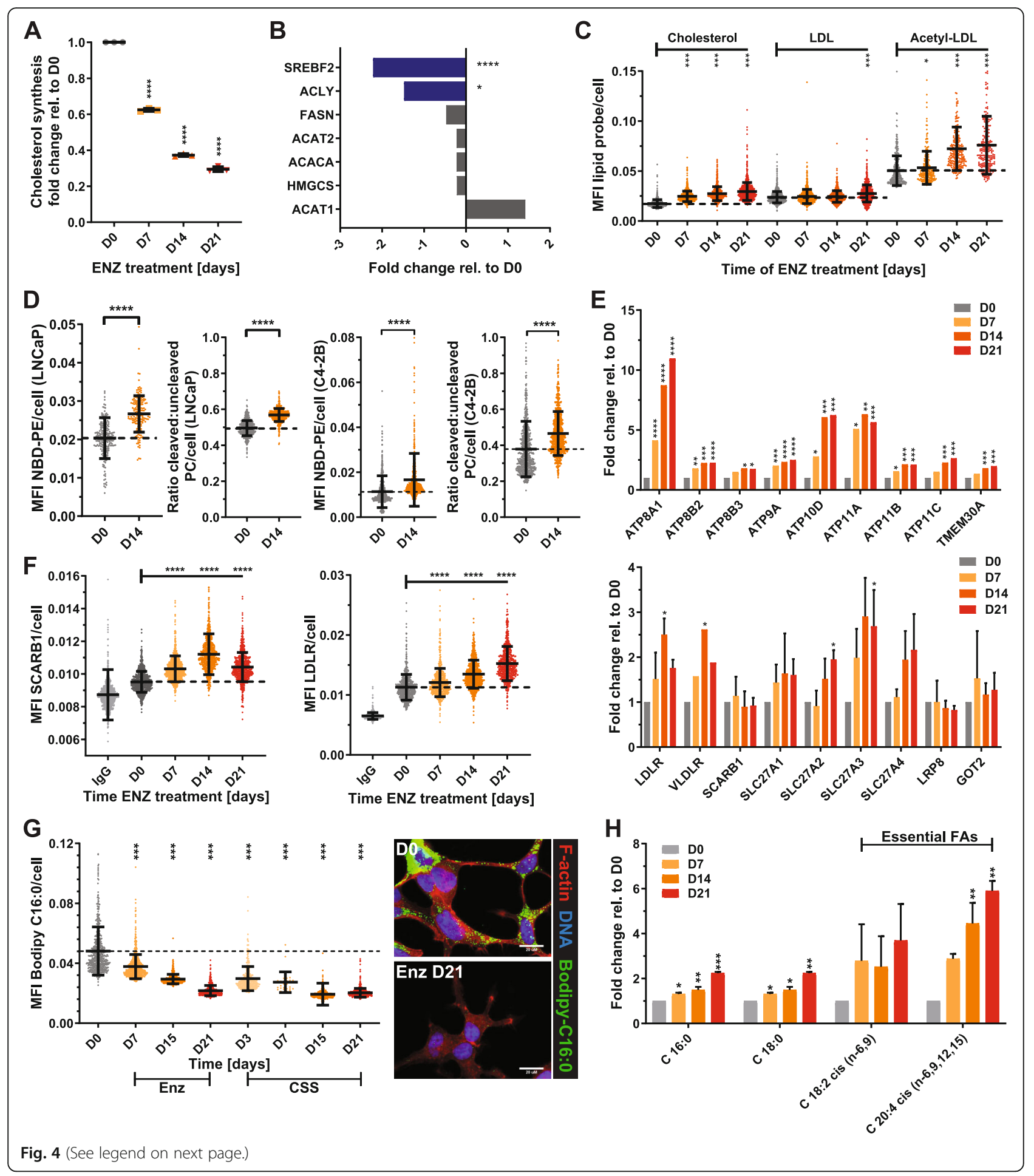




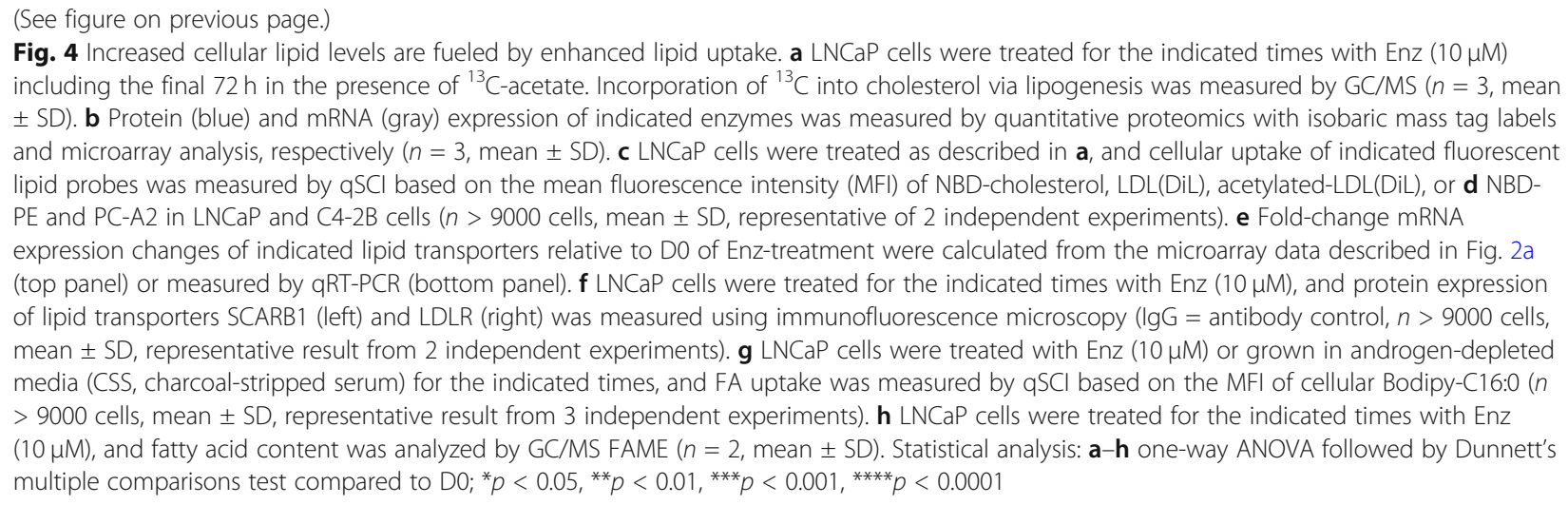

expression was reduced, providing further support that Enz-treated cells were increasingly acquiring PUFAs from uptake of serum-derived lipids (Fig. S4D) and by mobilizing PUFAs from lipid storage (e.g., from CEs and TAGs) with ongoing therapy (Figs. S3G and $\mathrm{S} 3 \mathrm{H})$.

\section{PUFA enrichment of membrane lipids is associated with increased lipid peroxidation and dependency on GPX4 activity}

High PUFA content of membrane phospholipids renders cells susceptible to oxidative stress through irondependent lipid peroxidation. In contrast, PUFAs stored as CEs and TAGs within lipid droplets are protected [48]. Excess amounts of PEs containing oxidized arachidonic acid (C20:4) or adrenic acid (C22:4) have been shown to trigger ferroptosis, a form of regulated, nonapoptotic cell death [12]. As shown above, Enz increased cellular levels of arachidonic acid (Fig. 4h) and PEs esterified with PUFAs (Fig. 5a). qSCI with the lipid peroxidation sensor C11-Bodipy and ratiometric analysis revealed that treatment of $\mathrm{LNCaP}$ and $\mathrm{C} 4-2 \mathrm{~B}$ cells for 14 days with Enz significantly increased lipid peroxidation (Fig. 5d). Glutathione peroxidase (GPX4) is critical for the reduction of toxic lipid hydroperoxides to their corresponding non-deleterious alcohols at the expense of reduced glutathione and thereby prevents cellular damage and induction of ferroptosis. LNCaP, C4-2B, DuCaP, and 22RV1 cells treated with Enz or CSS displayed hypersensitivity to the GPX4 inhibitor RSL3 as indicated by significantly increased cell death (Fig. 5e and S5D). Thus, several AR-positive PCa cell lines demonstrated increased dependency on GPX4 activity in response to ATTs, a characteristic previously shown to be associated with drug-induced persister cells in other types of cancer in response to different therapies [9, 49]. Cytotoxicity of RSL3 was completely suppressed by antioxidant and lipid peroxide scavenger Trolox, a vitamin $\mathrm{E}$ derivative, and ferroptosis inhibitor Ferrostatin-1 (Figs. 5e and S5D), confirming ferroptosis as the major cell death mechanism. Enhanced GPX4 dependence was detected as early as 4-8 days after commencement of ATTs (Enz and ADT) in LNCaP and C4-2B cells (Fig. $\mathrm{S} 5 \mathrm{E}$ ) and was fully reverted when cells were given a therapy holiday for three or more days (data not shown). Consistent with altered GPX4 dependence, mRNA levels of several genes involved in lipid peroxidation, ferroptosis, and redox homeostasis, including GPX4 and the recently identified ferroptosis suppressor protein 1 (FSP1/ AIFM2) [10, 11], were significantly altered by Enz (Fig. $5 \mathrm{f}$ ). In addition, Nrf2 (NFE2L2) is a master regulator of the antioxidant response [50], and several of its target genes that regulate iron and glutathione metabolism were also upregulated by Enz (Fig. 5f). Gene ontology analysis of protein expression changes (Fig. S1B) showed significant enrichment of pathways related to oxidative stress and selenocysteine metabolism which is an amino acid critical for the antioxidant function of selenoproteins (e.g., glutathione peroxidases, thioredoxin reductases, iodothyronine deiodinases, Fig. S5F).

Next, we tested if the above processes associated with lipid remodeling are critical mechanisms that underpin the development of GPX4 dependence in PCa persister cells. Indeed, acquisition of ferroptosis hypersensitivity, as measured by RSL3-induced cell death, was significantly suppressed in LNCaP (Fig. 5g) and C4-2B cells (data not shown) when Enz was combined with nontoxic doses of inhibitors directed against lipase activity (Orlistat and CAY10499) [51] or FA delta-6 desaturation (SC26196) [52]. Co-inhibition of the critical lipogenesis transcription factor SREBF1 with fatostatin [53] or ACACA (acetyl-CoA to malonyl-CoA conversion) and SCD1 (FA desaturation) with dual inhibitor TOFA [54] showed no or only limited reduction in ferroptosis sensitivity (Fig. $5 \mathrm{~g}$ ). The high protective efficiency of broad spectrum lipase inhibitor CAY10499 and FADS2 desaturase inhibitor SC26196 against RSL3-induced cell death in ENZ-treated LNCaP cells was comparable to 


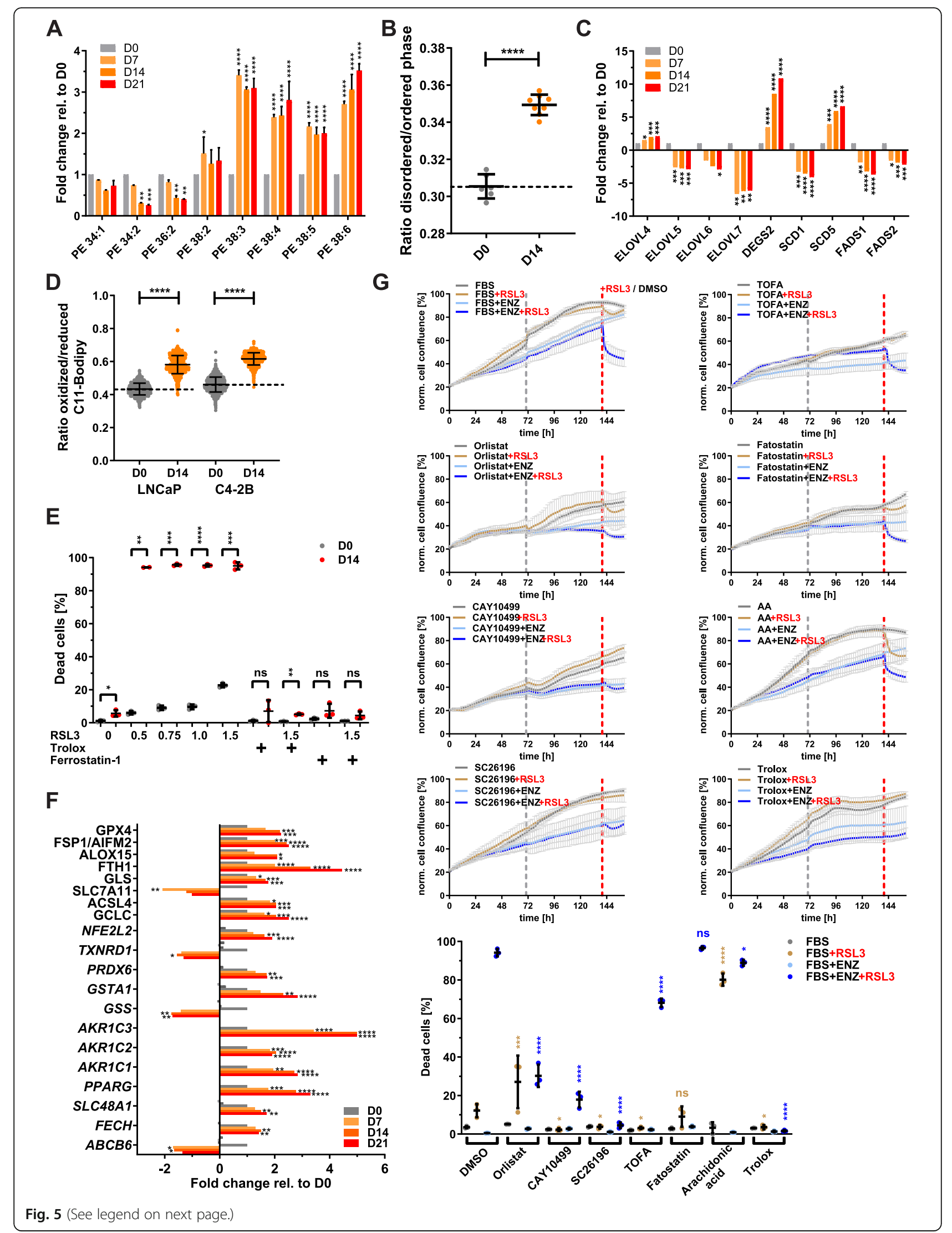


(See figure on previous page.)

Fig. 5 Therapy-induced lipid remodeling leads to increased membrane PUFA levels and lipid peroxidation and underpins GPX4 dependence. a LNCaP cells were treated for the indicated times with Enz $(10 \mu M)$, and lipids were analyzed by LC/MS. Individual sum compositions of phosphatidylethanolamine are shown $(n=2$, mean $\pm \mathrm{SD})$. $\mathbf{b} \mathrm{LNCaP}$ cells were treated as in $\mathbf{a}$, and membrane fluidity was measured by qSCl based on the mean fluorescence intensity (MFI) of Di-4-ANEPPDHQ ( $n=8$ wells, $\sim 750$ cells/well, mean \pm SD, representative results from 2 independent experiments). c Relative mRNA expression changes of indicated lipid remodeling enzymes (elongases and desaturases) were calculated from the microarray data described in Fig. 2a. $\mathbf{d}$ LNCaP cells were treated for the indicated times with Enz $(10 \mu \mathrm{M})$, and lipid peroxidation was measured by qSCl based on the mean fluorescence intensity (MFI) of Bodipy-C11 $(n>9000$ cells, mean \pm SD, representative results from 2 independent experiments). e LNCaP cells were cultured in growth media without (D0) or with Enz (10 $\mu \mathrm{M}, \mathrm{D} 14)$ for 14 days including the final $24 \mathrm{~h}$ in the presence of DMSO (0) or the indicated concentrations (0.5-1.5 $\mu \mathrm{M})$ of GPX4 inhibitor RSL3. Lipid peroxide scavenger Trolox (100 $\mu \mathrm{M})$ and ferroptosis inhibitor Ferrostatin-1 $(10 \mu \mathrm{M})$ were used as controls to demonstrate cell death through ferroptosis. The percentage of dead cells was calculated based on qSCl of Hoechst 3342 (total cell count) and propidium iodide (dead cells) staining ( $n=3$ wells/treatment with > 4000 cells/well, representative result of 3 independent experiments). $\mathbf{f}$ Relative mRNA expression changes of indicated key factors of ferroptosis and glutathione homeostasis were calculated from the microarray data described in Fig. 2a. $\mathbf{g}$ LNCaP cells seeded in a 96-well plate were co-treated with the indicated compounds (orlistat $10 \mu \mathrm{M}$, CAY10499 $17.5 \mu \mathrm{M}$, SC26196 $30 \mu \mathrm{M}$, TOFA $2.5 \mu \mathrm{M}$, fatostatin $5 \mu \mathrm{M}$, arachidonic acid (AA) $20 \mu \mathrm{M}$, Trolox $100 \mu \mathrm{M}$ ) in the absence (FBS) or presence of Enz (10 $\mu \mathrm{M}, \mathrm{FBS}+\mathrm{ENZ})$, and cell confluence was measured every $2 \mathrm{~h}$ by live cell imaging with an IncuCyte system $(n=3$, mean \pm SD). The gray dotted line indicates the time point of media change. After 7 days of co-treatment, cells were treated with vehicle control (DMSO/FBS) or RSL3 $(1.25 \mu \mathrm{M})$ to assess GPX4 dependence by monitoring changes to cell confluence for another day. The red dotted line indicates the start of RSL3 treatment. Cell death was then measured by qSCl (bottom graph) as described in (e; $n=3$ wells/treatment with $>4000$ cells/well, representative result of 2 independent experiments). Statistical analysis: a two-way ANOVA followed by Tukey's multiple comparisons test relative to D0; $\mathbf{b}-\mathbf{f}$ oneway ANOVA followed by Dunnett's multiple comparisons test compared to D0 or FBS+RSL3 (g, light brown labels) and FBS+ENZ+RSL3 (g, blue labels); ns, not significant, ${ }^{*} p<0.05,{ }^{* *} p<0.01$, ${ }^{* * *} p<0.001$, ${ }^{* * * *} p<0.0001$

that of Trolox (Fig. 5g) and Ferrostatin-1 (data not shown), suggesting that the co-inhibited processes are fundamental for developing Enz-induced GPX4 dependence. Similar results were observed when LNCaP cells were cultured in CSS, though the effect and efficacy of some co-treatments were different when compared to direct inhibition of AR with Enz, including that of fatostatin (Fig. S5G), suggesting that changes to the media composition (e.g., free FA levels, Fig. S4D) by charcoal stripping might have confounding effects. In support of this, supplementation of growth media with the PUFA arachidonic acid strongly intensified RSL3 sensitivity in parental, non-ATT treated cells (Fig. $5 \mathrm{~g}$ ), whereas reduced serum concentrations were protective (data not shown). This suggested a functional link of PUFA uptake mechanisms with ferroptosis sensitivity. Thus, interference with lipid supply and lipid remodeling pathways that were altered by ATTs (Enz and ADT) efficiently suppressed the acquisition of ferroptosis hypersensitivity. This demonstrated that therapy-induced GPX4 dependence can be modulated by rationally informed co-treatments targeting lipid remodeling and by altering PUFA and antioxidant levels of serum.

\section{Discussion}

Despite initial tumor regression following targeted cancer therapies, development of drug resistance and disease progression ultimately leading to patient death remain major clinical challenges of many pharmacological interventions in various types of cancer, including PCa. Previous studies of resistance to androgen receptor-targeted therapies (ATTs) in PCa analyzed models after extensive treatment periods (months to years) with fully developed resistance as indicated by reactivated cell proliferation $[6,8,33,34]$. Different to these studies, we hypothesized that delineating early events of ATT-induced metabolic reprogramming might provide novel co-targets that could extend the efficacy of ATTs, thereby delaying therapy failure and disease progression to currently incurable CRPC and Enz-resistant CRPC.

Our longitudinal study of the early adaptive response to androgen deprivation therapy (ADT) and Enz (0-21 days) in PCa cells made several key discoveries related to reprogrammed lipid metabolism (summarized in Fig. 6). Moreover, our work provided several novel potential targets for intervention strategies to combat ATT resistance that needs pre-clinical evaluation. Importantly, our study is first in class to demonstrate that therapy-induced lipid remodeling and lipid supply plasticity are critical mechanisms that underpin GPX4 dependence and ferroptosis hypersensitivity in persister cells. Thus, our findings might have wider implications for our understanding of early events of therapy resistance since GPX4 dependence has been reported in several types of cancer in response to different targeted therapies $[9,49]$.

Consistent with previous studies that showed that subpopulations of breast and lung cancer cells, called drug-induced persister cells, entered a quiescent state in response to anti-cancer treatments $([9,49]$ and reviewed in [55]), our functional studies showed that ATT-induced cellular quiescence was characterized by decreased proliferation, ATP production, and mitochondrial activity (Figs. 2c, S2C, and S2D). Previous studies have also reported a quiescence state of surviving cells after 3 weeks of androgen deprivation $[6$, 


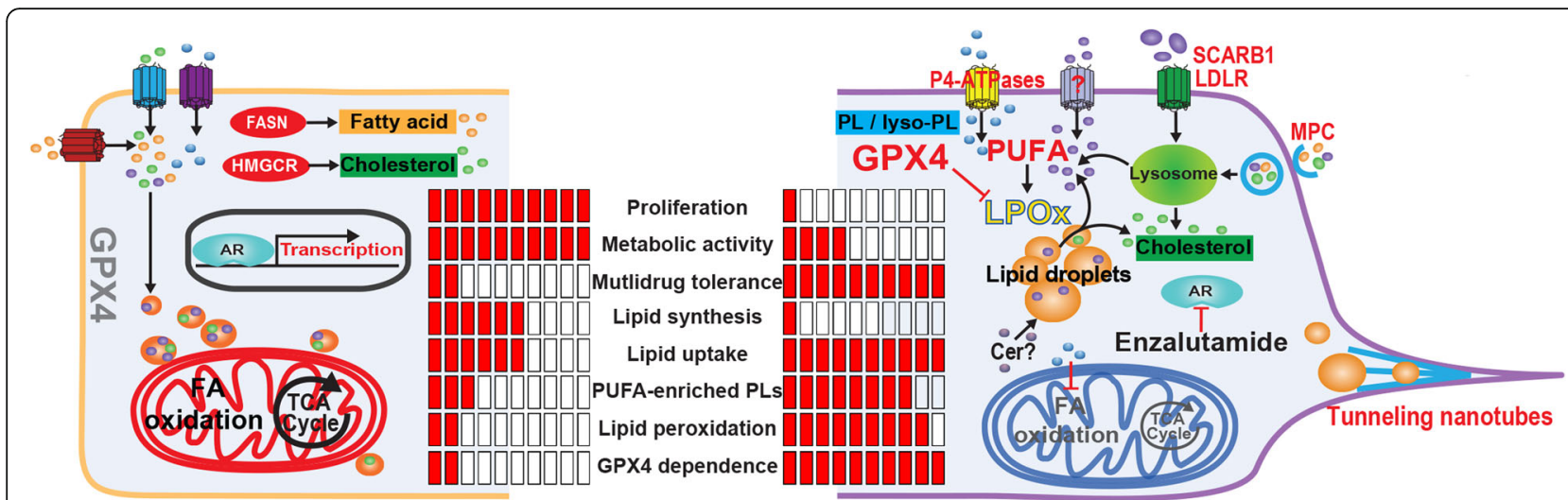

Fig. 6 Graphic representation of changes to lipid supply and lipid remodeling that underpin therapy-induced multidrug tolerance and ferroptosis hypersensitivity in PCa cells. In the androgen-stimulated, proliferative state (left), the cellular lipid pool is fueled by both de novo lipogenesis (DNL) of cholesterol (green) and FAs (orange) and lipid uptake. Mitochondrial FA oxidation and TCA cycle activity provide energy and precursor for biomass synthesis. In the therapy-induced quiescent state, DNL is reduced, whereas lipid uptake through cargo-selective (lipid transporters) and non-selective transport mechanisms (tunneling nanotubes and macropinocytosis, MPC) is enhanced. Therapy-induced lipid remodeling includes mobilization of storage lipids (CEs and TAGs) through lipases, increased levels of all major phospholipid species and their enrichment with PUFAs (purple), leading to increased membrane fluidity, lipid peroxidation (LPOX), and GPX4 dependence. Increased and decreased activities are highlighted in red and gray, respectively. Cer, ceramides

33, 34] and re-initiation of proliferation after $\sim 6$ months of treatment [6].

Unexpectedly, in light of cellular quiescence and low bioenergetic activity, Enz-induced remodeling of all major lipid classes (an energy-intensive process) was characterized by substantial accumulation of phospholipids and depletion of typical storage lipids (TAGs and CEs, Figs. 3 and S3) resident in lipid droplets (LDs). Yet, LD number and size (Fig. 3b) were increased. Therapyinduced LD formation in the context of growth inhibition has been previously demonstrated in cancer cells in response to oncogenic signaling inhibition [56]; however, the lipid composition of LDs was not delineated. It was recently shown that ceramides are converted to acylceramides via DGAT2 and stored in lipid droplets [57]. While DGAT1 and DGAT2 are both involved in TAG synthesis, DGAT2 also drives acylceramide synthesis and subsequent storage in lipid droplets. DGAT2 expression was upregulated in Enz-treated cells while DGAT1 was downregulated (Table S1), suggesting that increased acylceramide synthesis and lipid droplet storage caused the measured changes to lipid droplet morphometry. Ceramide metabolism was also highlighted by our comparative gene signature analysis (Fig. S1C). Given the heterogeneous nature of lipid droplets within cell populations [58] and that their accumulation has recently been described as a mechanism of drug resistance in colorectal cancer [59], renal cell carcinoma [56], and breast cancer cell lines [60], further investigations into lipid droplet biology under therapy stress are warranted.

Stable isotope carbon tracing revealed little glycolytic contribution to FA and cholesterol de novo synthesis (data not shown) and a 70\% reduction of cholesterol synthesis from acetate-derived carbon (Fig. 4a). While lipogenesis can be fueled by other carbon sources (glutamine and FAs), enhanced lipid synthesis from alternative fuels was not supported by the measured bioenergetic, redox, and mitochondrial activity statuses (Fig. 2c). Moreover, the transcriptome and proteome changes of Enz-treated LNCaP cells collectively indicated a reduction in oxidative phosphorylation (Fig. $2 \mathrm{~b}$ ) and, more importantly, a decrease of multiple key enzymes of de novo FA and cholesterol biosynthesis (Fig. $4 \mathrm{~b})$. These results were consistent with reduced toxicity of several lipogenesis inhibitors (Figs. 2d and S2G). Instead, therapy-induced lipid remodeling and increased lipid content was fueled by enhanced lipid uptake via cargo-selective (transporters, Fig. 4c-f) and nonselective uptake mechanisms (macropinocytosis and TNTs, Figs. S4E and S4F). We frequently observed mitochondria and lipid droplets within Enz-induced TNTs (Fig. S4F), suggesting that these conduits of intercellular transport might contribute considerable amounts of lipid biomass. Similarly, macropinocytosis of cell debris including membrane components have been previously shown to significantly contribute to the cellular lipid pool [45]. Notably, the lipid supply switch to uptake excluded uptake of saturated FAs as indicated by reduced uptake of C16:0-Bodipy after extended ATT treatments (Enz and ADT, Fig. 4g), indicating that other uptake mechanisms or transporters were responsible for the increased cellular levels of saturated FAs and PUFAs. Together, these results demonstrate that ATTs induced a switch of lipid supply from a mix of de novo synthesis and exogenous sources to predominantly uptake of exogenous lipids via multiple mechanisms. Thus, lipid 
supply plasticity is a novel metabolic mechanism associated with ATT resistance development in $\mathrm{PCa}$, and enhanced lipid scavenging can serve purposes other than biomass and energy production to support malignant cell proliferation. It is therefore critical to acknowledge that lipid supply plasticity during the development of ATT resistance limits the therapeutic window of individual treatments directed against either uptake or synthesis.

Only recently has attention been given to lipid uptake and to map the lipid transporter landscape in cancer cells [42, 61], including our own work in PCa [16]. The observed Enz-induced increase in protein expression of both LDLR and SCARB1 along with increased transcript levels of several other lipid transporters (Figs. 4e, f) supported the functional measurements obtained by qSCI of fluorescent lipid probes. They were concordant with previously reported transcriptional changes observed in our LNCaP tumor xenograft progression model in which recurring and CRPC tumors expressed higher levels of several lipid transporters and contained higher lipid content, including PUFAs, compared to tumors resected before castration [32]. Furthermore, increased expression of several lipid transporters were also observed in bone metastasis of CRPC patients (Fig. S4B, [16]); however, whether this is indirectly caused by the lipid-rich environment present in adult bone tissue (high adiposity) or directly by ATTs remains to be shown.

A major limitation of pharmacological targeting of PCa lipid supply is the scarcity of inhibitors and the incomplete understanding of mass contributions of different lipid supply pathways to the cellular lipid pool and their redundancies and cross-talk and compensatory capacities in response to reduced lipid synthesis, i.e., lipid supply plasticity. Lipogenesis on the other hand has remained a major target in cancer therapy, yielding an arsenal of inhibitors, unfortunately, with limited clinical success. There is increasing acknowledgement that various lipid scavenging pathways, including transportermediated uptake, tunneling nanotubes [46], lipidconjugated albumin uptake [62], and macropinocytosis [18], are critical supply routes of exogenous lipids fueling bioenergetic processes and biomass production that underpin tumor growth and survival. Recent studies of cancer cells expressing the lipogenic phenotype, including $\mathrm{PCa}$, estimated that $>70 \%$ of the lipid-derived carbon biomass is derived from uptake and only $30 \%$ from synthesis based on carbon tracing of serum-derived free FAs $[63,64]$. However, these estimates are limited by the fact that the serum lipidome is highly complex and that $>95 \%$ of serum FAs are acyl conjugates across all lipid classes, including TAGs, CEs, and phospholipids. Thus, the carbon contribution from the collective uptake of all lipid classes to lipid biomass in proliferating cancer cells is likely to be even higher than the above estimates. However, due to above reasons, the delineation of the mass contribution of individual uptake mechanisms will be technically challenging.

Despite these current limitations, we showed that blocking cholesterol uptake via NPC1 inhibition with U18666A (Fig. S2H) is a promising strategy to exploit the increased demand of ATT-treated PCa cells for exogenous cholesterol (Fig. 4c). Cholesterol is a critical storage lipid (as acyl-ester in lipid droplets) and serves as a precursor for the steroidogenic pathway in which testosterone can be endogenously synthesized in PCa tumors [65] and that activation of the steroidogenic pathway is an ATT-induced adaptive response contributing to CRPC progression despite castrate levels of circulating androgens [66-68]. Additionally, non-esterified, membrane cholesterol is shown to affect lipid raft composition which may influence oncogenic signaling [69], further highlighting the critical dependence of PCa cells on cholesterol for growth and survival, particularly under therapy stress. However, targeting cholesterol supply as a therapeutic strategy in PCa solely focused on the inhibition of de novo cholesterol synthesis, i.e., inhibition of the early steps of the mevalonate pathway (HGMCR with statins) [70]. Thus, co-targeting of lysosomal processing of exogenous cholesterol with U18666A and synthesis pathways with statins in the context of ATTs may prove a potent strategy that targets aforementioned aspects of cholesterol metabolism as well as sensitizes to ferroptosis through loss of intermediates of the mevalonate synthesis pathway (i.e., isopentenyl pyrophosphate and farnesyl diphosphate) that are critical for GPX4 [11] and FSP1 activities [13, 14]. Oxidative stress induction by co-targeting the mevalonate pathway and the glutathione pool via cystine import inhibition has recently been proven successful in causing profound tumor cell death in pancreatic cancer [71].

In addition to Enz-enhanced lipid uptake and content, our work described extensive lipid remodeling that was characterized by increased FA elongation and desaturation (Fig. 5a) of phospholipids. Both processes have previously been associated with $\mathrm{PCa}$ incidence and aggressiveness [72, 73] and have been reported to promote survival in therapy-resistant ovarian cancer stem cells [74]. Phospholipids containing long-chain FAs along with membrane-bound cholesterol are critical for membrane stabilization and are involved in lipid raft formation where oncogenic growth signaling occurs $[69,73]$. Enz-induced high PUFA levels in phospholipids were associated with increased membrane fluidity (Fig. 5b) which is thought to play a role in reduced sensitivity to chemotherapy reagents (i.e., multidrug tolerance, Fig. 2d) [75] and increases permeability and cell mobility and facilitates membrane-centered biological processes 
(vesicle formation, membrane fusion, cell-cell interaction, modulation of membrane enzymes, receptors, and transporters) [76]. Increased PUFA content of phospholipids does, however, generate a metabolic vulnerability [48]: increased oxidative damage through reactive oxygen and nitrogen species (ROS/RNS)-induced lipid peroxidation (Fig. 5d). Recent work showed that exogenous PUFAs can sensitize triple negative breast cancer to ferroptosis [77]. If not enzymatically repaired by GPX4 at the expense of reduced glutathione, accumulation of lipid hydroperoxides can lead to cell death via ferroptosis. Consistent with this, Enz-treated PCa cells showed reduced reductive power and hypersensitivity to selective GPX4 inhibitor, RSL3 (Fig. 5e). ADT and Enz-induced sensitivity to GPX4 inhibition of PCa cells was reminiscent of lapatinib- and vemurafenib-induced drug tolerance and emergence of "persister cells" in melanoma, breast, lung, and ovarian cancer [9] and therapyresistance-associated high-mesenchymal state cancer cells [78]. It was speculated that GPX4 dependence in these therapy-induced states relied on the FA composition of the lipid bilayers. Our results of therapy-induced lipid remodeling causing PUFA enrichment provide first-in-class evidence to support this hypothesis. More importantly, the diversity of targeted therapies causing this shared GPX4 dependence suggests that lipid remodeling leading to PUFA enrichment of phospholipids might be a common mechanism associated with therapy resistance that could be pursued as therapeutic target in multiple types of cancer. However, the treatment window of therapy-induced ferroptosis hypersensitivity is likely to be restricted to the low cycling, quiescence phase [9] because high membrane PUFA levels sensitize to ferroptosis [77] and would limit proliferation rates [48] due to the increased lipid peroxidation stress generated by higher metabolic activities and associated ROS production. Such stress would be further aggravated by higher demands in reducing power (NADPH) for the recycling of oxidized glutathione and de novo lipid synthesis.

Therefore, impeding the acquisition of the therapyinduced persister cell phenotype by co-targeting one or several lipid remodeling processes might be a more promising strategy that might extend the efficacy of existing cancer therapies and delay resistance than combatting fully developed and often complex resistance mechanisms. By using enhanced GPX4 dependence (RSL3 sensitivity) as a biomarker of the persister cell phenotype, we demonstrated that co-targeting of several different processes of lipid remodeling protected ATTtreated PCa cells from ferroptosis hypersensitivity (Figs. $5 \mathrm{~g}$ and $\mathrm{S} 5 \mathrm{G})$. In particular, the broad spectrum lipase inhibitors orlistat and CAY10499 [51] and FADS2 delta-6 desaturase inhibitor SC26196 [52] displayed high protective efficiencies against RSL3-induced cell death in
ENZ-treated LNCaP cells when compared to Trolox. This demonstrated that therapy-induced GPX4 dependence can be modulated by rationally informed cotreatments targeting lipid remodeling. Whether suppression of therapy-induced GPX4 dependence alters the course of ATT resistance remains to be shown. We noted that mRNA expression of the majority of FA elongases and desaturases, including SCD1 and FADS2, were reduced after Enz treatment for 7-21 days (Fig. 5c). Yet, inhibitors against these enzymes efficiently suppressed Enz-induced ferroptosis hypersensitivity when co-treated for 7 days (Figs. 5g and S5G). Thus, the different treatment periods might explain the divergence between inhibitor activity and target expression, i.e., Enz induces a time-dependent switch from endogenous PUFA synthesis by FA elongation and desaturation to enhanced uptake of exogenous PUFAs as demonstrated for uptake of other lipid species (Figs. 4c, d). Notably, FADS2 activity has been recently implicated in cancer plasticity $[74,79]$ and FADS2 inhibition with SC26196 impaired cancer stemness, $\mathrm{NF \kappa B}$ signaling, and tumor initiation capacity [74]. Our work indicates a potential functional link between FADS2 activity and therapy-induced GPX4 dependence that warrants further investigations. Whether co-targeting of lipid remodeling in combination with ATTs ultimately delays resistance development is a priority for further studies. Furthermore, the ferroptosis altering effects of PUFA and antioxidant supplementation might have implications for $\mathrm{PCa}$ patients undergoing ATT treatment, i.e., changing the duration until therapy failure, that need evaluation.

It is clear from this work that, despite its numerous discoveries, there are many unanswered questions regarding mechanisms of therapy-induced lipid remodeling (e.g., if changes to lipidome are reverted upon acquisition of resistance) and how this process can be harnessed to extend efficacies of existing, clinically proven therapies. The most prevalent are confirmation of the PUFA-enriched phospholipid phenotype in other types of cancer displaying therapy-induced GPX4 dependence $[9,78]$, investigating if dietary aspects like PUFA consumption and antioxidant supplementation affect resistance development and pre-clinical testing of co-therapies against lipid remodeling to combat ATT resistance development and disease progression to currently lethal CRPC.

\section{Conclusions}

Breast, melanoma, lung, and ovarian cancer treated with different therapies develop GPX4 dependence and ferroptosis hypersensitivity, which are metabolic hallmarks of persister cells, an early, drug-induced state that is associated with acquired therapy resistance and tumor relapse. Our study in prostate cancer in response to androgen-targeted therapies demonstrated that GPX4 
dependence and ferroptosis hypersensitivity of persister cells are associated with extensive lipid remodeling, including enhanced lipid uptake and PUFA enrichment of membrane lipids (Fig. 6). It showed that lipase and fatty acid desaturase activities are critical for persister cells to develop GPX4 dependence. Since enhanced GPX4 dependence is an adaptive state shared by several types of cancer in response to different therapies, our discoveries might have universal implications for our understanding of metabolic events that underpin acquired resistance and inform novel co-treatments against tumor relapse.

\section{Supplementary information}

Supplementary information accompanies this paper at https://doi.org/10. 1186/s40170-020-00217-6.

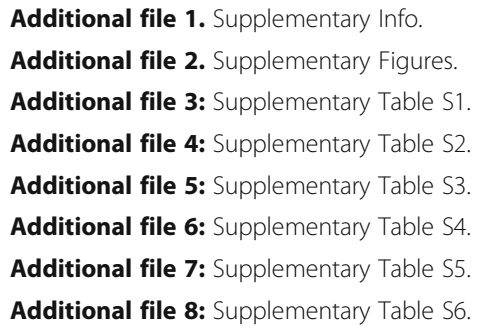

\section{Abbreviations}

ADT: Androgen deprivation therapy; AR: Androgen receptor; ATT: Androgen receptor-targeted therapy; CE: Cholesterol ester; Cer: Ceramide; CRPC: Castration-resistant prostate cancer; CSS: Charcoal-stripped serum; DNL: De novo lipogenesis; Enz: Enzalutamide; ESI-MS: Electrospray ionization mass spectrometry; FA: Fatty acid; FDR: False discovery rate; GC/MS: Gas chromatography tandem mass spectrometry; GSVA: Gene set variation analysis; INT: Intact; LC/MS: Liquid chromatography tandem mass spectrometry; LD: Lipid droplet; LDL: Low density lipoprotein; LPC: Lysophosphatidylcholine; LPOx: Lipid peroxidation; MDT: Multidrug tolerance; MFI: Mean fluorescence intensity; MPC: Macropinocytosis; NAD: Nadir; PC: Phosphatidylcholine; PCa: Prostate cancer; PE: Phosphatidylethanolamine; PG: Phosphatidylglycerol; PL: Phospholipid; PS: Phosphatidylserine; PUFA: Polyunsaturated fatty acid; qSCl: Quantitative single-cell imaging; RNS: Reactive nitrogen species; ROS: Reactive oxygen species; SM: Sphingomyelin; TAG: Triacylglycerol; TNT: Tunneling nanotube

\section{Acknowledgements}

We would like to thank Prof Dr Bart Ghesquière, Mr. Abel Acosta Sanchez, and the Metabolomics Expertise Center from the department of Oncology (KU Leuven) and the Center for Cancer Biology (CCB, VIB) for the ${ }^{13} \mathrm{C}$ carbon tracing analyses. We thank the Proteomics Core Facility and Translational Cell Imaging Queensland located at the Translational Research Institute for their technical assistance.

\section{Authors' contributions}

Conception and design: Martin C Sadowski, Kaylyn D Tousignant. Development of methodology: Martin C Sadowski, Kaylyn D Tousignant, Anja Rockstroh, Berwyck LJ Poad, Rajesh Gupta, Stephen J Blanksby, Reuben SE Young. Acquisition of data: Kaylyn D Tousignant, Martin C Sadowski, Ali Talebi, Anja Rockstroh. Analysis and interpretation of data (i.e., RNAseq analysis, computational analysis): Kaylyn D Tousignant, Martin C Sadowski, Anja Rockstroh, Atefeh Taherian Fard, Melanie L Lehman, Chenwei Wang, Berwyck ᄂ Poad, Rajesh Gupta, Reuben SE Young, Ali Talebi, Johan V Swinnen, Colleen C Nelson. Writing, review, and/or revision of the manuscript: Kaylyn D Tousignant, Martin C Sadowski, Melanie L Lehman, Stephen J Blanksby, Colleen C Nelson. The author(s) read and approved the final manuscript.

\section{Funding}

This study was supported by the Movember Foundation and the Prostate Cancer Foundation of Australia through a Movember Revolutionary Team Award, a Chenhall Research Award, and the Australian Government Department of Health. The majority of this work was conducted at the Translational Research Institute which is supported by a grant from the Australian Government.

\section{Availability of data and materials}

Normalized gene expression data of the microarray experiment have been deposited in NCBI's Gene Expression Omnibus (GEO) and are accessible through GEO Series accession number GSE143408. The proteomics dataset, lipid mass spectrometry data, and CellProfiler analysis pipelines are available from the corresponding author on request.

Ethics approval and consent to participate

Not applicable.

\section{Consent for publication}

Not applicable

\section{Competing interests}

The authors declare that they have no competing interest.

\section{Author details}

${ }^{1}$ Australian Prostate Cancer Research Centre - Queensland, Institute of Health and Biomedical Innovation, School of Biomedical Sciences, Faculty of Health, Queensland University of Technology (QUT), Princess Alexandra Hospital,

Translational Research Institute, Brisbane, Australia. ${ }^{2}$ Cancer \& Ageing Research Program, Institute of Health and Biomedical Innovation, School of Biomedical Sciences, Faculty of Health, Queensland University of Technology (QUT), Translational Research Institute, Brisbane, Australia. ${ }^{3}$ Central Analytical Research Facility, Institute for Future Environments, Queensland University of Technology, Brisbane, Australia. ${ }^{4}$ Department of Oncology, Laboratory of Lipid Metabolism and Cancer, LKI Leuven Cancer Institute, KU Leuven-University of Leuven, Leuven, Belgium. ${ }^{5}$ Vancouver Prostate Centre, Department of Urologic Sciences, University of British Columbia, Vancouver, Canada.

Received: 16 January 2020 Accepted: 8 May 2020

Published online: 19 June 2020

\section{References}

1. Litwin MS, Tan $\mathrm{H}$. The diagnosis and treatment of prostate cancer: a review. JAMA. 2017;317(24):2532-42.

2. Heinlein CA, Chang C. Androgen receptor in prostate cancer. Endocr Rev. 2004:25(2):276-308.

3. Lonergan PE, Tindall DJ. Androgen receptor signaling in prostate cancer development and progression. J Carcinogenesis. 2011;10:20.

4. Risbridger GP, et al. Breast and prostate cancer: more similar than different. Nat Rev Cancer. 2010;10(3):205-12.

5. Dutt SS, Gao AC. Molecular mechanisms of castration-resistant prostate cancer progression. Future Oncol. 2009;5(9):1403-13.

6. Lu S, Tsai SY, Tsai M-J. Molecular mechanisms of androgen-independent growth of human prostate cancer LNCaP-Al Cells1. Endocrinology. 1999; 140(11):5054-9.

7. Höti N, et al. Proteomics analyses of prostate cancer cells reveal cellular pathways associated with androgen resistance. Proteomics. 2017;17(6):10. 1002/pmic.201600228.

8. Kregel $\mathrm{S}$, et al. Acquired resistance to the second-generation androgen receptor antagonist enzalutamide in castration-resistant prostate cancer. Oncotarget. 2016;7(18):26259-74.

9. Hangauer MJ, et al. Drug-tolerant persister cancer cells are vulnerable to GPX4 inhibition. Nature. 2017;551:247.

10. Yang WS, et al. Regulation of ferroptotic cancer cell death by GPX4. Cell. 2014;156(1-2):317-31.

11. Seibt TM, Proneth B, Conrad M. Role of GPX4 in ferroptosis and its pharmacological implication. Free Radic Biol Med. 2019;133:144-52.

12. Kagan VE, et al. Oxidized arachidonic and adrenic PEs navigate cells to ferroptosis. Nat Chem Biol. 2017;13(1):81-90. 
13. Bersuker K, et al. The CoQ oxidoreductase FSP1 acts parallel to GPX4 to inhibit ferroptosis. Nature. 2019.

14. Doll S, et al. FSP1 is a glutathione-independent ferroptosis suppressor. Nature. 2019.

15. Locke JA, et al. Steroidogenesis inhibitors alter but do not eliminate androgen synthesis mechanisms during progression to castration-resistance in LNCaP prostate xenografts. J Steroid Biochem Mol Biol. 2009;115(3-5):126-36.

16. Tousignant KD, et al. Lipid uptake is an androgen-enhanced lipid supply pathway associated with prostate cancer disease progression and bone metastasis; 2019. p. molcanres.1147.2018.

17. Desir $\mathrm{S}$, et al. Tunneling nanotube formation is stimulated by hypoxia in ovarian cancer cells. Oncotarget. 2016;7(28):43150-61

18. Finicle BT, Jayashankar V, Edinger AL. Nutrient scavenging in cancer. Nat Rev Cancer. 2018;18(10):619-33.

19. Jaishy B, Abel ED. Lipids, lysosomes, and autophagy. J Lipid Res. 2016;57(9):1619-35.

20. Schmittgen TD, Livak KJ. Analyzing real-time PCR data by the comparative C(T) method. Nat Protoc. 2008;3(6):1101-8.

21. Smyth GK. Limma: linear models for microarray data; 2005. p. 397-420.

22. Hänzelmann S, Castelo R, Guinney J. GSVA: gene set variation analysis for microarray and RNA-Seq data. BMC Bioinformatics. 2013;14(1):7.

23. Subramanian A, et al. Gene set enrichment analysis: a knowledge-based approach for interpreting genome-wide expression profiles. Proc Natl Acad Sci U S A. 2005;102(43):15545-50.

24. Eden E, et al. GOrilla: a tool for discovery and visualization of enriched GO terms in ranked gene lists. BMC Bioinformatics. 2009;10:48.

25. Kolde R, Vilo J. GOsummaries: an R package for visual functional annotation of experimental data. F1000Res. 2015;4:574.

26. Li S, et al. Activation of MAPK signaling by CXCR7 leads to enzalutamide resistance in prostate cancer. 2019;79(10):2580-92.

27. Li Q, et al. Linking prostate cancer cell AR heterogeneity to distinct castration and enzalutamide responses. Nat Commun. 2018;9(1):3600.

28. Rajan P, et al. Next-generation sequencing of advanced prostate cancer treated with androgen-deprivation therapy. Eur Urol. 2014;66(1):32-9.

29. Levrier $\mathrm{C}$, et al. Denhaminols $\mathrm{A}-\mathrm{H}$, dihydro-beta-agarofurans from the endemic Australian rainforest plant Denhamia celastroides. J Nat Prod. 2015; 78(1):111-9.

30. Carpenter $A E$, et al. CellProfiler: image analysis software for identifying and quantifying cell phenotypes. Genome Biol. 2006;7(10):R100.

31. Butte W. Rapid method for the determination of fatty acid profiles from fats and oils using trimethylsulphonium hydroxide for transesterification. Chromatogr A. 1983;261:142-5.

32. Locke JA, et al. Arachidonic acid activation of intratumoral steroid synthesis during prostate cancer progression to castration resistance. Prostate. 2010; 70(3):239-51.

33. Yu P, et al. Androgen-independent LNCaP cells are a subline of LNCaP cells with a more aggressive phenotype and androgen suppresses their growth by inducing cell cycle arrest at the G1 phase. Int J Mol Med. 2017;40(5):1426-34.

34. $\mathrm{Xu} \mathrm{G}$, et al. Characterization of the small RNA transcriptomes of androgen dependent and independent prostate cancer cell line by deep sequencing. PLoS ONE. 2010;5(11):e15519.

35. Pfeiffer MJ, et al. Steroidogenic enzymes and stem cell markers are upregulated during androgen deprivation in prostate cancer. Mol Med. 2011;17(7-8):657-64

36. Pfeffer SR. NPC intracellular cholesterol transporter 1 (NPC1)-mediated cholesterol export from lysosomes. J Biol Chem. 2019;294(5):1706-9.

37. Swinnen $\mathrm{JV}$, et al. Androgens stimulate fatty acid synthase in the human prostate cancer cell line LNCaP. Cancer Res. 1997;57(6):1086-90.

38. Swinnen JV, et al. Androgens markedly stimulate the accumulation of neutral lipids in the human prostatic adenocarcinoma cell line LNCaP. Endocrinology. 1996;137(10):4468-74.

39. Greenspan P, Mayer E, Fowler S. Nile red: a selective fluorescent stain for intracellular lipid droplets. J Cell Biol. 1985;100(3):965-73.

40. Maxfield FR, Wüstner D. Analysis of cholesterol trafficking with fluorescent probes. Methods Cell Biol. 2012;108:367-93.

41. Christie WW. Rapid separation and quantification of lipid classes by high performance liquid chromatography and mass (light-scattering) detection. J Lipid Res. 1985;26(4):507-12.

42. Iglesias-Gato $D$, et al. The proteome of prostate cancer bone metastasis reveals heterogeneity with prognostic implications. Clin Cancer Res. 2018.

43. Ikonen E. Cellular cholesterol trafficking and compartmentalization. Nat Rev Mol Cell Biol. 2008;9(2):125-38.
44. Goldstein JL, Anderson RG, Brown MS. Receptor-mediated endocytosis and the cellular uptake of low density lipoprotein. CIBA Found Symp. 1982;92:77-95.

45. Kim SM, et al. PTEN deficiency and AMPK activation promote nutrient scavenging and anabolism in prostate cancer cells. Cancer Discov. 2018;8(7):866-83.

46. Astanina $\mathrm{K}$, et al. Lipid droplets as a novel cargo of tunnelling nanotubes in endothelial cells. Sci Rep. 2015;5:-11453.

47. Agbaga M-P, et al. Role of Stargardt-3 macular dystrophy protein (ELOVL4) in the biosynthesis of very long chain fatty acids. Proc Natl Acad Sci U S A. 2008;105(35):12843-8

48. Bailey AP, et al. Antioxidant role for lipid droplets in a stem cell niche of Drosophila. Cell. 2015:163(2):340-53.

49. Ramirez $M$, et al. Diverse drug-resistance mechanisms can emerge from drug-tolerant cancer persister cells. Nat Commun. 2016;7:-10690.

50. Dodson M, Castro-Portuguez R, Zhang DD. NRF2 plays a critical role in mitigating lipid peroxidation and ferroptosis. Redox Biol. 2019;23:101107.

51. Iglesias J, et al. Simplified assays of lipolysis enzymes for drug discovery and specificity assessment of known inhibitors. J Lipid Res. 2016:57(1):131-41.

52. Obukowicz MG, et al. Novel, selective delta6 or delta5 fatty acid desaturase inhibitors as antiinflammatory agents in mice. J Pharmacol Exp Ther. 1998; 287(1):157-66.

53. Li X, et al. Fatostatin displays high antitumor activity in prostate cancer by blocking SREBP-regulated metabolic pathways and androgen receptor signaling. Mol Cancer Ther. 2014;13(4):855-66.

54. Mason P, et al. SCD1 inhibition causes cancer cell death by depleting mono-unsaturated fatty acids. PLoS One. 2012;7(3):e33823.

55. Vallette FM, et al. Dormant, quiescent, tolerant and persister cells: four synonyms for the same target in cancer. Biochem Pharmacol. 2018.

56. Lue HW, et al. Metabolic reprogramming ensures cancer cell survival despite oncogenic signaling blockade. Genes Dev. 2017;31(20):2067-84.

57. Senkal CE, et al. Ceramide is metabolized to acylceramide and stored in lipid droplets. Cell Metab. 2017;25(3):686-97.

58. Petan $T$, Jarc $E_{1}$ Jusović M. Lipid droplets in cancer: guardians of fat in a stressful world. Molecules. 2018;23(8):1941.

59. Cotte AK, et al. Lysophosphatidylcholine acyltransferase 2-mediated lipid droplet production supports colorectal cancer chemoresistance. Nat Commun. 2018:9(1):322

60. Hultsch S, et al. Association of tamoxifen resistance and lipid reprogramming in breast cancer. BMC Cancer. 2018;18(1):850.

61. Iglesias-Gato D, et al. The proteome of primary prostate cancer. Eur Urol. 2016;69(5):942-52.

62. Bern $\mathrm{M}$, et al. The role of albumin receptors in regulation of albumin homeostasis: implications for drug delivery. J Control Release. 2015;211:144-62.

63. Hosios AM, et al. Amino acids rather than glucose account for the majority of cell mass in proliferating mammalian cells. Dev Cell. 2016;36(5):540-9.

64. Balaban $\mathrm{S}$, et al. Extracellular fatty acids are the major contributor to lipid synthesis in prostate cancer. Mol Cancer Res. 2019.

65. Locke JA, et al. Androgen levels increase by intratumoral de novo steroidogenesis during progression of castration-resistant prostate cancer. Cancer Res. 2008;68(15):6407-15.

66. Dillard PR, Lin M-F, Khan SA. Androgen-independent prostate cancer cells acquire the complete steroidogenic potential of synthesizing testosterone from cholesterol. Mol Cell Endocrinol. 2008;295(1-2):115-20.

67. Leon CG, et al. Alterations in cholesterol regulation contribute to the production of intratumoral androgens during progression to castration-resistant prostate cancer in a mouse xenograft model. Prostate. 2010:70(4):390-400.

68. Ghayee HK, Auchus RJ. Basic concepts and recent developments in human steroid hormone biosynthesis. Rev Endocr Metab Disord. 2007:8(4):289-300.

69. Zhuang $L$, et al. Cholesterol targeting alters lipid raft composition and cell survival in prostate cancer cells and xenografts. J Clin Investig. 2005;115(4):959-68.

70. Gordon JA, et al. Oral simvastatin administration delays castration-resistant progression and reduces intratumoral steroidogenesis of LNCaP prostate cancer xenografts. Prostate Cancer Prostatic Dis. 2016;19(1):21-7.

71. McGregor GH, et al. Targeting the metabolic response to statin-mediated oxidative stress produces a synergistic antitumor response. Cancer Res. 2020;80(2):175-88

72. Peck B, et al. Inhibition of fatty acid desaturation is detrimental to cancer cell survival in metabolically compromised environments. Cancer Metabol. 2016:4:6.

73. Tamura K, et al. Novel lipogenic enzyme ELOVL7 is involved in prostate cancer growth through saturated long-chain fatty acid metabolism. Cancer Res. 2009;69(20):8133-40. 
74. Li J, et al. Lipid desaturation is a metabolic marker and therapeutic target of ovarian cancer stem cells. Cell Stem Cell. 2017;20(3):303-14 e5.

75. Corsetto $P$, et al. $\omega-3$ long chain polyunsaturated fatty acids as sensitizing agents and multidrug resistance revertants in cancer therapy. Int J Mol Sci. 2017;18(12):2770.

76. van Meer G, Voelker DR, Feigenson GW. Membrane lipids: where they are and how they behave. Nature reviews. Mol Cell Biol. 2008;9(2):112-24.

77. Beatty $\mathrm{A}$, et al. Conjugated linolenic fatty acids trigger ferroptosis in triplenegative breast cancer; 2019. p. 556084.

78. Viswanathan VS, et al. Dependency of a therapy-resistant state of cancer cells on a lipid peroxidase pathway. Nature. 2017;547:453.

79. Vriens $\mathrm{K}$, et al. Evidence for an alternative fatty acid desaturation pathway increasing cancer plasticity. Nature. 2019;566(7744):403-6.

\section{Publisher's Note}

Springer Nature remains neutral with regard to jurisdictional claims in published maps and institutional affiliations.

Ready to submit your research? Choose BMC and benefit from:

- fast, convenient online submission

- thorough peer review by experienced researchers in your field

- rapid publication on acceptance

- support for research data, including large and complex data types

- gold Open Access which fosters wider collaboration and increased citations

- maximum visibility for your research: over $100 \mathrm{M}$ website views per year

At $\mathrm{BMC}$, research is always in progress.

Learn more biomedcentral.com/submissions 